\title{
Polynomial solutions for arbitrary higher spin Dirac operators
}

\author{
D. Eelbode $^{*} \quad$ T. Raeymaekers ${ }^{\dagger}$
}

\begin{abstract}
In a series of recent papers, we have introduced higher spin Dirac operators, which are far-reaching generalisations of the classical Dirac operator. Whereas the latter acts on spinor-valued functions, the former acts on functions taking values in arbitrary irreducible half-integer highest weight representations for the spin group. In this paper, we describe a general procedure to decompose the polynomial kernel spaces for these operators in irreducible summands for the regular action of the spin group. We will do this in an inductive way, making use of twisted higher spin operators.
\end{abstract}

Keywords: higher spin; Dirac operators; representation, spin group, Clifford analysis

\section{Introduction}

This paper deals with higher spin Dirac operators (HSD operators for short), which are elliptic first-order differential operators generalising the classical Dirac operator from physics, in the sense that they act on functions $f(x)$ which are defined on $\mathbb{R}^{m}$ and take their values in arbitrary irreducible finite-dimensional modules for the spin group or its orthogonal Lie algebra $\mathfrak{s o}(m)$. In the framework of theoretical physics, these operators (acting on higher spin fields) are usually defined in terms of the abstract index notation (tensor notation), but there exists a nice alternative to study these operators within the setting of Clifford analysis. This is a function theory generalising both classical complex analysis and harmonic analysis on $\mathbb{R}^{m}$, see e.g. $[1,11,15,16]$. The advantage of the latter framework, as opposed to the one involving abstract indices, lies in the fact that one can make use of explicit realisations and computations in several vector variables to study function theoretical properties of the HSD operators. Apart from the references mentioned earlier, centered around the classical Dirac operator, we also mention e.g. [5, 6], in which the Rarita-Schwinger operator was generalised and studied, and the more recent papers $[3,9]$ in which further generalisations were considered. The aim of the present paper is to study the vector space of polynomial solutions for arbitrary HSD operators as a spin group module. This is a non-trivial question, which was already treated for the RaritaSchwinger operators $\mathcal{R}_{l_{1}}$ and $\mathcal{Q}_{l_{1}, l_{2}}$ in respectively [5] and [2], but still remains an open

\footnotetext{
${ }^{*}$ Department of Mathematics and Computer Science, University of Antwerp, Campus Middelheim, G-Building, Middelheimlaan 1, 2020 Antwerpen, Belgium, email: David.Eelbode@ua.ac.be

${ }^{\dagger}$ Clifford Research Group, Department of Mathematical Analysis, Ghent University, Galglaan 2, 9000 Ghent, Belgium, email: tr@cage.ugent.be (corresponding author)
} 
question for the most general operator $\mathcal{Q}_{l_{1}, \ldots, l_{k}}$. The main problem lies in the fact that the HSD operators stand in sharp contrast to the classical Dirac operator. Whereas the space of polynomial solutions for the latter defines a model for an irreducible spin representation, solution spaces for the former are highly reducible and need to be decomposed into several summands.

In order to tackle this problem, we will first introduce basic concepts from both Clifford analysis and representation theory in section 2, allowing us to define the HSD operators in full generality. In section 3, we will turn our attention to two types of higher spin operators, the higher spin twistor operators (HST operators for short) and the higher spin Dirac operators, realising them as generators (among other operators) for the transvector algebra $Z(\mathfrak{o s p}(1,2 k+2), \mathfrak{o s p}(1,2 k))$ and proving a few results which are crucial for what follows. In section 4 , we will then define the so-called twisted version for these HSD operators, and introduce a special class of solutions for HSD operators, in order to prepare ourselves for the main topic of this article. In section 5, an inductive argument will be used to formulate a conjecture describing arbitrary solutions (reducing it to a combinatorial problem), and in section 6 we will illustrate how this can be verified up to order 3 using a computer package.

\section{Notations and definitions}

In this paper, we will constantly work with invariant operators acting on functions taking values in irreducible representations for the spin group (cfr. infra) or its Lie algebra $\mathfrak{s o}(m)$. A useful model for both this Lie group and its Lie algebra can be given in terms of the (real or complex) Clifford algebra $\mathbb{R}_{m}$ and $\mathbb{C}_{m}=\mathbb{R}_{m} \otimes \mathbb{C}$. Both algebras are defined in terms of the standard orthonormal basis $\left\{e_{j}: 1 \leq j \leq m\right\}$ for the underlying vector space $\mathbb{R}^{m}$ by means of the multiplication rules $e_{i} e_{j}+e_{j} e_{i}=-2 \delta_{i j}$. The space of (real) bivectors is defined as

$$
\mathbb{R}_{m}^{(2)}:=\operatorname{span}_{\mathbb{R}}\left\{e_{i j}:=e_{i} e_{j}: 1 \leq i<j \leq m\right\} \subset \mathbb{R}_{m},
$$

and one has that $\mathfrak{s o}(m) \cong \mathbb{C}_{m}^{(2)}=\mathbb{R}_{m}^{(2)} \otimes \mathbb{C}$, where the Lie bracket is given by the standard commutator bracket. The (real) spin group, for which $\mathfrak{s o}(m)$ defines the (complex) Lie algebra, is given by even products of unit vectors:

$$
\operatorname{Spin}(m):=\left\{\prod_{j=1}^{2 k} \omega_{j}: \omega_{j} \in S^{m-1}\right\},
$$

where $S^{m-1} \subset \mathbb{R}^{m}$ stands for the unit sphere. Arbitrary finite-dimensional representations $\mathbb{V}_{\lambda}$ for the spin group or its Lie algebra are then characterised by their highest weight, containing coordinates $\lambda=\left(l_{1}, \ldots, l_{n}\right)$ with respect to the standard basis $\left\{L_{j}: 1 \leq j \leq n\right\}$ for the dual space $\mathfrak{h}^{*}$. Here, $\mathfrak{h}$ denotes a Cartan subalgebra for the algebra $\mathfrak{s o}(m)$ with $m=2 n$ or $m=2 n+1$. Note that we will restrict ourselves to odd dimensions from now on, which means that $\mathfrak{s o}(m)=B_{n}$ in Dynkin's notation. 
Remark 1. Although the results are not conceptually different for even dimensions, there are notational complications arising from the fact that the space of Dirac spinors decomposes into even and odd Weyl spinors. All the results in this paper are also true in case of an even dimension as well, as one can also consider the entire (reducible) space of Dirac spinors in even dimension. However, if one wants irreducible representations, each representation has to be split up according to parity of the underlying Weyl spinors.

Let us then introduce models for arbitrary irreducible half-integer highest weight modules. First of all, we have the spinor space $\mathbb{S}$, characterised by its highest weight $\left(\frac{1}{2}, \ldots, \frac{1}{2}\right)$, which can be realised as the vector space $\mathbb{S} \cong \mathbb{S}_{2 n+2}^{ \pm}$. The action is then given by standard multiplication $B \cdot \psi:=B \psi$, for all $B \in \mathfrak{s o}(m)$ and $\psi \in \mathbb{S}$. The (massless) Dirac operator, lying at the very core of Clifford analysis, can now be defined as the conformally invariant elliptic differential operator acting on functions taking values in the spinor space $\mathbb{S}$. This operator is given by $\partial_{x}:=\sum_{j} e_{j} \partial_{x_{j}}$, and factorises the Laplace operator in the sense that $\partial_{x}^{2}=-\Delta_{x}$. This implies that the vector space containing polynomial solutions for $\partial_{x}$ can be seen as a subspace of the space of $\mathbb{C}_{m}$-valued harmonic polynomials. We will often use homogeneous polynomials, for which we introduce the following notation: homogeneous polynomials of degree $h$ in a vector variable $x$ will be denoted by $\mathcal{P}_{h}\left(\mathbb{R}^{m}, \mathbb{C}\right)$. Next, we also need dummy variables $u_{j} \in \mathbb{R}^{m}$. We will denote the Euler operator (resp. Dirac operator and Laplace operator) in terms of the vector variable $u_{j}$ by means of $\mathbb{E}_{j}$ (resp. $\partial_{j}$ and $\Delta_{j}$ ), and the Euclidean inner product on $\mathbb{R}^{m}$ by $\langle\cdot, \cdot\rangle$. Also, define the angular momentum operator by $L_{i j}^{x}=x_{i} \partial_{x_{j}}-x_{j} \partial_{x_{i}}$. The following result refines the fact that spherical harmonics define an irreducible module under the action of the orthogonal Lie algebra:

Proposition 1 (e.g. [7]). The vector space of $l_{1}$-homogeneous monogenic polynomials, defined by means of

$$
\mathcal{M}_{l_{1}}\left(\mathbb{R}^{m}, \mathbb{S}\right):=\left\{M_{l_{1}}\left(u_{1}\right) \in \mathcal{P}_{l_{1}}\left(\mathbb{R}^{m}, \mathbb{C}\right) \otimes \mathbb{S}: \partial_{1} M_{l_{1}}\left(u_{1}\right)=0\right\},
$$

gives a model for the irreducible $\mathfrak{s o}(m)$-module with highest weight $\left(l_{1}+\frac{1}{2}, \frac{1}{2}, \ldots, \frac{1}{2}\right)$ under the (derived) regular $d L$-action, for all $e_{i j} \in \mathfrak{s o}(m)$ given by

$$
d L\left(e_{i j}\right) \cdot M_{l_{1}}\left(u_{1}\right):=\left.\frac{d}{d t}\right|_{t=0} e^{t e_{i j}} M_{l_{1}}\left(e^{-t e_{i j}} u_{1} e^{t e_{i j}}\right)=\left(e_{i j}-2 L_{i j}^{u_{1}}\right) M_{l_{1}}\left(u_{1}\right) .
$$

This can be generalised to arbitrary (half-integer) highest weights of the form

$$
\lambda^{\prime}:=\left(l_{1}, \ldots, l_{k}\right)^{\prime}=\left(l_{1}+\frac{1}{2}, l_{2}+\frac{1}{2}, \ldots, l_{k}+\frac{1}{2}, \frac{1}{2}, \ldots, \frac{1}{2}\right),
$$

where $l_{1} \geq l_{2} \geq \ldots \geq l_{k}>0$ (the so-called dominant weight condition), and where we have introduced a short-hand notation for half-integer highest weights (the prime denotes the Cartan product with the spinor space). To do so, one only needs to consider functions $f\left(u_{1}, \ldots, u_{k}\right)$ in the dummy vector variables $u_{j} \in \mathbb{R}^{m}$, taking values in the spinor space and satisfying a certain system of differential equations. For notational ease, we will denote $\left(u_{1}, \ldots, u_{k}\right)$ by $u_{(k)}$. Then one has (see e.g. $\left.[7,15]\right)$ : 
Proposition 2. A polynomial $S_{\lambda}\left(u_{(k)}\right) \in \mathcal{P}_{\lambda}\left(\mathbb{R}^{k m}, \mathbb{C}\right) \otimes \mathbb{S}$ is simplicial monogenic of degree $\lambda=\left(l_{1}, \ldots, l_{k}\right)$ in the variables $\left(u_{1}, \ldots, u_{k}\right)$ if it satisfies the following equations:

$$
\begin{aligned}
& \mathbb{E}_{j} S_{\lambda} \quad=l_{j} S_{\lambda} \quad \forall 1 \leq j \leq k \\
& \partial_{j} S_{\lambda} \quad=0 \quad \forall 1 \leq j \leq k \\
& \left\langle u_{i}, \partial_{j}\right\rangle S_{\lambda}=0 \quad \forall 1 \leq i<j \leq k .
\end{aligned}
$$

In case $l_{1} \geq l_{2} \geq \ldots \geq l_{k}$, the vector space $\mathcal{S}_{\lambda}\left(\mathbb{R}^{k m}, \mathbb{S}\right)$ containing $\lambda$-homogeneous simplicial monogenics defines a model for the irreducible $\mathfrak{s o}(m)$-module with highest weight $\lambda^{\prime}$ under the (derived) regular action, for all $e_{i j} \in \mathfrak{s o}(m)$ given by

$$
\begin{aligned}
d L\left(e_{i j}\right) \cdot S_{\lambda}\left(u_{(k)}\right) & :=\left.\frac{d}{d t}\right|_{t=0} e^{t e_{i j}} S_{\lambda}\left(e^{-t e_{i j}} u_{1} e^{t e_{i j}}, \ldots, e^{-t e_{i j}} u_{k} e^{t e_{i j}}\right) \\
& =\left(e_{i j}-2 \sum_{p=1}^{k} L_{i j}^{u_{p}}\right) S_{\lambda}\left(u_{(k)}\right) .
\end{aligned}
$$

In [7], it was shown that the irreducible finite-dimensional representation $\mathcal{S}_{\lambda}$, with highest weight $\lambda^{\prime}$, is generated by the highest weight vector

$$
P_{\lambda}\left(u_{(k)}\right)=\left\langle u_{1}, \mathfrak{f}_{1}\right\rangle^{l_{1}-l_{2}}\left\langle u_{1} \wedge u_{2}, \mathfrak{f}_{1} \wedge \mathfrak{f}_{2}\right\rangle^{l_{2}-l_{3}} \cdots\left\langle u_{1} \wedge \cdots \wedge u_{k}, \mathfrak{f}_{1} \wedge \cdots \wedge \mathfrak{f}_{k}\right\rangle^{l_{k}} I,
$$

where each of these inner products is defined by means of

$$
\begin{aligned}
\left\langle u_{1} \wedge \cdots \wedge u_{k}, \mathfrak{f}_{1} \wedge \cdots \wedge \mathfrak{f}_{k}\right\rangle & =\operatorname{det}\left(\begin{array}{ccc}
\left\langle u_{1}, \mathfrak{f}_{1}\right\rangle & \cdots & \left\langle u_{1}, \mathfrak{f}_{k}\right\rangle \\
\vdots & \ddots & \vdots \\
\left\langle u_{k}, \mathfrak{f}_{1}\right\rangle & \cdots & \left\langle u_{k}, \mathfrak{f}_{k}\right\rangle
\end{array}\right) \\
& =\sum_{\sigma \in S_{k}} \operatorname{sgn}(\sigma)\left\langle u_{\sigma(1)}, \mathfrak{f}_{1}\right\rangle \cdots\left\langle u_{\sigma(k)}, \mathfrak{f}_{k}\right\rangle
\end{aligned}
$$

with $S_{k}$ the symmetric group in $k$ elements, and where $I=\prod_{j=1}^{n} \mathfrak{f}_{j} \mathfrak{f}_{j}^{\dagger}$ with

$$
\left(\mathfrak{f}_{j}, \mathfrak{f}_{j}^{\dagger}\right):=\left(\frac{e_{j}-i e_{j+n}}{2},-\frac{e_{j}+i e_{j+n}}{2}\right)
$$

defines the Witt basis. We then have that

$$
\mathcal{S}_{\lambda}=\operatorname{Span}_{\mathbb{C}}\left\{s P_{\lambda}\left(s^{*} u_{1} s, \ldots, s^{*} u_{k} s\right): s \in \operatorname{Spin}(m)\right\} .
$$

One can now consider smooth functions $f\left(x ; u_{(k)}\right) \in \mathcal{C}^{\infty}\left(\mathbb{R}^{m}, \mathcal{S}_{\lambda}\right)$ taking values in the module $\mathcal{S}_{\lambda}$. This means that for each $x \in \mathbb{R}^{m}$ fixed, the resulting polynomial in the dummy variables $u_{j}$ satisfies the requirements from the proposition above. On these functions, two types of conformally invariant differential operators can be defined: generalisations of the Dirac operator (HSD operators) and higher spin twistor operators (HST operators). The former preserve the values and are elliptic, whereas the latter map functions taking values in $\mathcal{S}_{\lambda}$ to functions taking values in $\mathcal{S}_{\mu}$ with $\lambda \neq \mu$. Our main interest is the following operator: 
Definition 1. For an arbitrary half-integer highest weight $\lambda^{\prime}$, the associated HSD operator $\mathcal{Q}_{\lambda}$ is given by

$$
\mathcal{Q}_{\lambda}:=\prod_{j=1}^{k}\left(1+\frac{u_{j} \partial_{j}}{2 \mathbb{E}_{j}+m-2}\right) \partial_{x}: \mathcal{C}^{\infty}\left(\mathbb{R}^{m}, \mathcal{S}_{\lambda}\right) \rightarrow \mathcal{C}^{\infty}\left(\mathbb{R}^{m}, \mathcal{S}_{\lambda}\right) .
$$

Note that the product is understood to be ordered, with indices increasing from left to right, and that the Euler operator in the variable $u_{j}$ in the denominator acts as the constant $l_{j}$. Also note that in case $\lambda^{\prime}=\left(l_{1}\right)^{\prime}$, which corresponds to higher-dimensional versions of the classical Rarita-Schwinger operator coming from physics [23], the operator is denoted by means of $\mathcal{R}_{l_{1}}$ rather than $\mathcal{Q}_{l_{1}}$.

Remark 2. We refer the reader to section 3 for the construction of these operators.

The true aim of this paper is to describe polynomial null solutions $f\left(x ; u_{(k)}\right)$ satisfying the equation $\mathcal{Q}_{\lambda} f\left(x ; u_{(k)}\right)=0$. To do so, we will make use of the twistor operators, whose existence is implied by Fegan's result and the theory of generalised Stein-Weiss gradients, see $[13,25]$.

\section{Higher spin operators}

For the detailed construction of HSD and HST operators, we refer to the recent article [12], but we will give a brief overview here as well. The idea is to construct these operators as generators of the transvector algebra $Z(\mathfrak{o s p}(1,2 k+2), \mathfrak{o s p}(1,2 k))$. The abstract definitions of such algebras can be found in e.g. [22, 26, 27]. We will recall the definitions in the present Clifford analysis context. First, we need the definition of the Lie superalgebra $\mathfrak{o s p}(1,2 k)$ as an operator subalgebra of the Clifford-Weyl algebra

$$
\mathcal{W} \otimes \mathbb{C}_{m}=\operatorname{Alg}_{\mathbb{R}}\left(u_{i j}, \partial_{u_{i j}}: 1 \leq i \leq k, 1 \leq j \leq m\right) \otimes \mathbb{C}_{m}
$$

Lemma 1. The orthosymplectic Lie superalgebra osp $(1,2 k)$ is the algebra generated by

$$
\mathfrak{o s p}(1,2 k)=\operatorname{Alg}\left(u_{1}, \ldots, u_{k}, \partial_{1}, \ldots, \partial_{k}\right),
$$

whereby the even Lie subalgebra $\mathfrak{s p}(2 k)$ is generated by

$$
\mathfrak{s p}(2 k)=\mathfrak{k}=\operatorname{Alg}\left(\left\langle\partial_{i}, \partial_{j}\right\rangle,\left\langle u_{i}, u_{j}\right\rangle,\left\langle u_{i}, \partial_{j}\right\rangle: 1 \leq i, j \leq k\right) .
$$

Let us introduce the subalgebra $\mathfrak{h} \subset \mathfrak{o s p}(1,2 k)$

$$
\mathfrak{h}=\operatorname{Alg}\left(\mathbb{E}_{i}+\frac{m}{2}: 1 \leq i \leq k\right)
$$

which serves as our Cartan subalgebra. One can now define a crucial object $p_{\mathfrak{o s p}(1,2 k)}$ inside the algebra $\mathcal{U}^{\prime}(\mathfrak{o s p}(1,2 k))$, which is defined as the tensor product of the universal enveloping algebra $\mathcal{U}(\mathfrak{o s p}(1,2 k))$ for the algebra $\mathfrak{o s p}(1,2 k)$ and the field $R(\mathfrak{h})$ of fractions in the Cartan subalgebra (this means that Euler operators will appear in the denominator). 
Definition 2. The extremal projector for the Lie algebra $\mathfrak{o s p}(1,2 k)$ is the uniquely defined element $p_{\mathfrak{o s p}(1,2 k)} \in \mathcal{U}^{\prime}(\mathfrak{o s p}(1,2 k))$ satisfying the requirements $p_{\mathfrak{o s p}(1,2 k)} \mathfrak{k}^{-}=\mathfrak{k}^{+} p_{\mathfrak{o s p}(1,2 k)}=0$ and $p_{\mathfrak{o s p}(1,2 k)}^{2}=p_{\mathfrak{o s p}(1,2 k)}$. The subalgebras $\mathfrak{k}^{ \pm}$are hereby defined as the subspaces containing positive (resp. negative) root vectors:

$$
\begin{aligned}
\mathfrak{k}^{+} & =\mathfrak{k}_{0}^{+} \oplus \mathfrak{k}_{1}^{+} \\
& =\operatorname{span}\left(\Delta_{a},\left\langle\partial_{a}, \partial_{b}\right\rangle,\left\langle u_{i}, \partial_{j}\right\rangle: 1 \leq i<j \leq k, 1 \leq a \neq b \leq k\right) \oplus \operatorname{span}\left(\partial_{a}: 1 \leq a \leq k\right) \\
\mathfrak{k}^{-} & =\mathfrak{k}_{0}^{-} \oplus \mathfrak{k}_{1}^{-} \\
& =\operatorname{span}\left(\left|u_{a}\right|^{2},\left\langle u_{a}, u_{b}\right\rangle,\left\langle u_{j}, \partial_{i}\right\rangle: 1 \leq i<j \leq k, 1 \leq a \neq b \leq k\right) \oplus \operatorname{span}\left(u_{a}: 1 \leq a \leq k\right) .
\end{aligned}
$$

Hereby, the subindex 0 and 1 refer to the even and odd part of $\mathfrak{o s p}(1,2 k)$ respectively.

For the explicit form for this extremal projection operator $p_{\mathfrak{0 s p}(1,2 k)}$ we refer to [12], as we will only need a simplified version here. The upshot is that the HSD operators are defined as the operators $p_{\mathfrak{o s p}(1,2 k)} \partial_{x}$, and the twistor operators as the operators $p_{\mathfrak{o s p}(1,2 k)}\left\langle\partial_{x}, \partial_{a}\right\rangle$, with $1 \leq a \leq k$. The twistor operators clearly lower the degree in one of the variables $u_{a}$, and preserve simplicial monogenicity of the values due to the properties of the extremal projector. As a matter of fact, since the operators $\Delta_{j},\left\langle\partial_{i}, \partial_{j}\right\rangle$ and $\partial_{j}$ commute with $\left\langle\partial_{x}, \partial_{a}\right\rangle$, it is enough to consider the extremal projection operator related to the subalgebra $\mathfrak{g l}(k) \subset \mathfrak{o s p}(1,2 k)$ for the twistor operators. Plugging in this expression, and omitting redundant factors, this leads to the following definitions:

Definition 3. For an arbitrary highest weight $\lambda^{\prime}=\left(l_{1}, \ldots, l_{k}\right)^{\prime}$ with $l_{k}>0$, one can define the HSD operators

$$
\mathcal{Q}_{\lambda}: \mathcal{C}^{\infty}\left(\mathbb{R}^{m}, \mathcal{S}_{\lambda}\right) \rightarrow \mathcal{C}^{\infty}\left(\mathbb{R}^{m}, \mathcal{S}_{\lambda}\right)
$$

The (reduced) explicit form for these operators is given by

$$
\mathcal{Q}_{\lambda}=p_{\mathfrak{o s p}(1,2 k)} \partial_{x}=\prod_{i=1}^{k}\left(1+\frac{u_{i} \partial_{i}}{m+2 \mathbb{E}_{i}-2 i}\right) \partial_{x},
$$

Note that the product in this equation is ordered (i increasing from left to right), as the factors do not commute. Also, in accordance with Remark 1, one has to mention that the HSD operators changes the parity of Weyl spinors.

Definition 4. For an arbitrary half-integer highest weight $\lambda=\left(l_{1}, \ldots, l_{k}\right)^{\prime}$ with $l_{k}>0$, one can define the HST operators

$$
\mathcal{T}_{\lambda}^{(j)}: \mathcal{C}^{\infty}\left(\mathbb{R}^{m}, \mathcal{S}_{\lambda}\right) \rightarrow \mathcal{C}^{\infty}\left(\mathbb{R}^{m}, \mathcal{S}_{l_{1}, \ldots, l_{j-1}, l_{j}-1, l_{j+1}, \ldots, l_{k}}\right)
$$

provided $l_{j}>l_{j+1}$. The upper index $(j)$ hereby refers to the variable in which the degree of homogeneity will decrease. Their explicit form is up to a constant given by

$$
\mathcal{T}_{\lambda}^{(j)}:=p_{\mathfrak{o s p}(1,2 k)}\left\langle\partial_{x}, \partial_{j}\right\rangle=\prod_{p=j+1}^{k}\left(1+\frac{1}{\mathbb{E}_{p}-\mathbb{E}_{j}+j-(p+1)}\left\langle u_{p}, \partial_{j}\right\rangle\left\langle u_{j}, \partial_{p}\right\rangle\right)\left\langle\partial_{j}, \partial_{x}\right\rangle,
$$


for $j<k$ and $\mathcal{T}_{\lambda}^{(k)}=\left\langle\partial_{k}, \partial_{x}\right\rangle$. Note that this product is also understood to be ordered, with increasing indices from left to right. The Euler operators in the denominator again automatically introduce constants $l_{j}$ in case a fixed $\lambda$ is chosen. Considering Remark 1 again, one has to mention that the HST operators preserve the parity of Weyl spinors.

Remark 3. Note that because of the Euler operators appearing in the explicit formulas, these operators are essentially independent of $\lambda$. This is why we will from now on also use the notations $\mathcal{T}^{(j)}:=\mathcal{T}_{\lambda}^{(j)}$ for the HST operators, and $\mathcal{Q}:=\mathcal{Q}_{\lambda}$ for HSD operators, unless it is essential to know what space the operators act on. This will considerably reduce the notation load.

In general, these operators have nice commutation relations, coming from the fact that they are generators of the transvector algebra mentioned earlier. We will explicitly prove the relations that will play a crucial role in what follows.

Lemma 2. For all $a<b$, we have the relation

$$
\mathcal{T}^{(b)} \mathcal{T}^{(a)}=\mathcal{T}^{(a)} \mathcal{T}^{(b)} \frac{\mathbb{E}_{a}-\mathbb{E}_{b}+b-a+1}{\mathbb{E}_{a}-\mathbb{E}_{b}+b-a},
$$

which means that HST operators commute up to a coefficient in $R(\mathfrak{h})$.

Proof. When expanding the product in the expression of $\mathcal{T}^{(a)}$, we get that

$$
\mathcal{T}^{(a)}=\left\langle\partial_{a}, \partial_{x}\right\rangle+\sum_{a<i_{1}<\cdots<i_{s} \leq k} \frac{\left\langle u_{i_{1}}, \partial_{a}\right\rangle\left\langle u_{i_{2}}, \partial_{i_{1}}\right\rangle \cdots\left\langle u_{i_{s}}, \partial_{i_{s-1}}\right\rangle\left\langle\partial_{i_{s}}, \partial_{x}\right\rangle}{\left(\mathbb{E}_{a}-\mathbb{E}_{i_{1}}+i_{1}-a\right) \cdots\left(\mathbb{E}_{a}-\mathbb{E}_{i_{s}}+i_{s}-a\right)} .
$$

Assume that $a<b$, then we notice that because of the properties of the extremal projector and the fact that $\left\langle\partial_{a}, \partial_{x}\right\rangle$ commutes with each factor of the projector in $\mathcal{T}^{(b)}$ in its simplest form (see Definition 4 ), we get that

$$
p_{\mathfrak{o s p}(1,2 k)}\left\langle\partial_{a}, \partial_{x}\right\rangle p_{\mathfrak{o s p}(1,2 k)}\left\langle\partial_{b}, \partial_{x}\right\rangle=p_{\mathfrak{o s p}(1,2 k)}\left\langle\partial_{a}, \partial_{x}\right\rangle\left\langle\partial_{b}, \partial_{x}\right\rangle
$$

Still keeping in mind that the extremal projector $p_{\mathfrak{o s p}(1,2 k)}$ has the property that

$$
p_{\mathfrak{o s p}(1,2 k)}\left\langle u_{j}, \partial_{i}\right\rangle=0
$$

and using (3) for all $i<j$, a straightforward calculation gives us

$$
p_{\mathfrak{o s p}(1,2 k)}\left\langle\partial_{b}, \partial_{x}\right\rangle p_{\mathfrak{o s p}(1,2 k)}\left\langle\partial_{a}, \partial_{x}\right\rangle=p_{\mathfrak{o s p}(1,2 k)}\left\langle\partial_{a}, \partial_{x}\right\rangle\left\langle\partial_{b}, \partial_{x}\right\rangle \frac{\mathbb{E}_{a}-\mathbb{E}_{b}+b-a+1}{\mathbb{E}_{a}-\mathbb{E}_{b}+b-a} .
$$

Thus, if $a \leq b$, by combining (4) and (5), we arrive at

$$
\mathcal{T}^{(b)} \mathcal{T}^{(a)}=\mathcal{T}^{(a)} \mathcal{T}^{(b)} \frac{\mathbb{E}_{a}-\mathbb{E}_{b}+b-a+1}{\mathbb{E}_{a}-\mathbb{E}_{b}+b-a},
$$

as was to be proved. 
Remark 4. In Lemma 2, by commuting, we merely mean that the expressions of the twistor operators commute up to a coefficient in $R(\mathfrak{h})$, as elements of the transvector algebra. When acting on functions, the operators on the LHS and RHS of (2) are in fact different operators, as they act on polynomials of a different degree of homogeneity. The same remark also holds for the following lemma.

Lemma 3. We have the relation

$$
\mathcal{T}^{(a)} \mathcal{Q}=\frac{m+\mathbb{E}_{a}-2 a}{m+\mathbb{E}_{a}-2 a+2} \mathcal{Q} \mathcal{T}^{(a)},
$$

meaning that HST operators and HSD operators commute up to a coefficient in $R(\mathfrak{h})$.

Proof. On the one hand we have that

$$
\mathcal{Q} \mathcal{T}^{(a)}=p_{\mathfrak{o s p}(1,2 k)} \partial_{x} p_{\mathfrak{o s p}(1,2 k)}\left\langle\partial_{a}, \partial_{x}\right\rangle=p_{\mathfrak{o s p}(1,2 k)} \partial_{x}\left\langle\partial_{a}, \partial_{x}\right\rangle,
$$

since $\left\langle u_{j}, \partial_{i}\right\rangle$ and $\partial_{x}$ commute, and $p_{\mathfrak{o s p}(1,2 k)}\left\langle u_{j}, \partial_{i}\right\rangle=0$ for all $i<j$. On the other hand, a straightforward calculation shows that

$$
\begin{aligned}
p_{\mathfrak{o s p}(1,2 k)}\left\langle\partial_{a}, \partial_{x}\right\rangle p_{\mathfrak{o s p}(1,2 k)} \partial_{x} & =p_{\mathfrak{o s p}(1,2 k)}\left\langle\partial_{a}, \partial_{x}\right\rangle \prod_{i=a}^{k}\left(1+\frac{u_{i} \partial_{i}}{m+2 \mathbb{E}_{i}-2 i}\right) \partial_{x} \\
& =\frac{m+\mathbb{E}_{a}-2 a}{m+\mathbb{E}_{a}-2 a+2} p_{\mathfrak{o s p}(1,2 k)}\left\langle\partial_{a}, \partial_{x}\right\rangle \partial_{x}
\end{aligned}
$$

again using the properties of the extremal projector.

\section{Recursively defined HSD operators}

The explicit form for the operator $\mathcal{Q}_{\lambda}$ in definition 1 was obtained in e.g. [9], using the notion of twisted Dirac operators. It is however also possible to define these operators through the action of twisted HSD operators, which has the advantage that they allow for a recursive definition. In [10], we have introduced the following definition, which is based on the fact that for $\lambda=\left(l_{1}, \ldots, l_{k}\right)$, one has that $\mathcal{S}_{\lambda} \subset \mathcal{S}_{l_{1}, \ldots, l_{k-1}} \otimes \mathcal{H}_{l_{k}}$.

Definition 5. Given an arbitrary highest weight $\lambda^{\prime}=\left(l_{1}, \ldots, l_{k}\right)^{\prime}$ with $k>1$, one can define the twisted HSD operator by means of

$$
\mathcal{Q}_{l_{1}, \ldots, l_{k-1}}^{T}:=\mathcal{Q}_{l_{1}, \ldots, l_{k-1}} \otimes 1: \mathcal{C}^{\infty}\left(\mathbb{R}^{m}, \mathcal{S}_{\lambda}\right) \rightarrow \mathcal{C}^{\infty}\left(\mathbb{R}^{m}, \mathcal{S}_{l_{1}, \ldots, l_{k-1}} \otimes \mathcal{H}_{l_{k}}\right)
$$

where $\mathcal{H}_{l_{k}}=\mathcal{P}_{l_{k}}\left(\mathbb{R}^{m}, \mathbb{C}\right) \cap$ ker $\Delta_{k}$ denotes the vector space of $l_{k}$-homogeneous harmonic (scalar-valued) polynomials in the variable $u_{k} \in \mathbb{R}^{m}$.

Remark 5. Note that this basically means that the twisted $H S D$ operator $\mathcal{Q}_{l_{1}, \ldots, l_{k-1}} \otimes \mathbf{1}$ is the HSD operator associated to a related highest weight acting on functions assuming the 'wrong' values. In Clifford analysis, this merely amounts to the difference between e.g. $\mathcal{R}_{l_{1}} f\left(x ; u_{1}\right)$ and $\mathcal{R}_{l_{1}} f\left(x ; u_{1}, u_{2}\right)$. Provided $f\left(x ; u_{1}\right)$ takes values in $\mathcal{M}_{l_{1}}$, the former defines an action of a true HSD operator, whereas the latter is a twisted HSD operator, acting on functions $f\left(x ; u_{1}, u_{2}\right)$ taking values in $\mathcal{S}_{l_{1}, l_{2}}$. 
Remark 6. From now on, we will almost never write these twisted HSD operators using the tensor product symbol. It will be clear from the context or explicitly mentioned when the 'twisted' version of an operator is used (like in the example above).

Despite the fact that the tensor product at the right hand side of the definition above becomes quite complicated in general (it is not even multiplicity-free, except for a few trivial cases), the image of the twisted version of the operator $\mathcal{Q}_{\lambda}$ acting on $\mathcal{S}_{\lambda}$-valued functions is contained in the sum of two submodules only (see [10]):

Proposition 3. Given an arbitrary highest weight $\lambda^{\prime}=\left(l_{1}, \ldots, l_{k}\right)^{\prime}$ with $k>1$, one has:

$$
\mathcal{Q}_{l_{1}, \ldots, l_{k-1}}^{T}: \mathcal{C}^{\infty}\left(\mathbb{R}^{m}, \mathcal{S}_{\lambda}\right) \rightarrow \mathcal{C}^{\infty}\left(\mathbb{R}^{m}, \mathcal{S}_{\lambda} \oplus \mathcal{S}_{\lambda-L_{k}}\right)
$$

More explicitly, the action on $\mathcal{S}_{\lambda}$-valued functions decomposes as follows:

$$
\mathcal{Q}_{l_{1}, \ldots, l_{k-1}}^{T}=\mathcal{Q}_{\lambda}+\frac{2}{2 l_{k}+m-2 k} \pi_{l_{1}, \ldots, l_{k-1}}\left[u_{k}\right] \mathcal{T}_{\lambda}^{(k)} .
$$

Hereby, the operator $\pi_{l_{1}, \ldots, l_{k-1}}$ has to be seen as the projection of the multiplication operator $u_{k}$ on the space $\mathcal{S}_{l_{1}, \ldots, l_{k-1}} \otimes \mathcal{H}_{l_{k}}$, i.e.

$$
\pi_{l_{1}, \ldots, l_{k-1}}\left[u_{k}\right]=\prod_{j=1}^{k-1}\left(1+\frac{u_{j} \partial_{j}}{2 l_{j}+m-2}\right) u_{k}: \mathcal{C}^{\infty}\left(\mathbb{R}^{m}, \mathcal{S}_{\lambda}\right) \rightarrow \mathcal{C}^{\infty}\left(\mathbb{R}^{m}, \mathcal{S}_{l_{1}, \ldots, l_{k-1}} \otimes \mathcal{H}_{l_{k}}\right)
$$

Remark 7. Note that the factor $\pi_{l_{1}, \ldots, l_{k-1}}\left[u_{k}\right]$ in the proposition is often referred to as the so-called embedding factor. This notation stems from the fact that decomposing tensor products like the one from above, i.e. $\mathcal{S}_{l_{1}, \ldots, l_{k-1}} \otimes \mathcal{H}_{l_{k}}$, consists of two tasks: first of all, one can make a list of all highest weights of irreducible modules which are included as a submodule. Secondly, for each of these submodules there is an embedding factor explicitly realising the way in which this submodule occurs inside the tensor product. For example, in abstract notation one has for all $l_{1}>0$ :

$$
\left(l_{1}\right) \otimes\left(\frac{1}{2}, \ldots, \frac{1}{2}\right) \cong\left(l_{1}\right)^{\prime} \oplus\left(l_{1}-1\right)^{\prime},
$$

whereas in Clifford analysis this becomes

$$
\mathcal{H}_{l_{1}}\left(\mathbb{R}^{m}, \mathbb{S}\right)=\mathcal{M}_{l_{1}}\left(\mathbb{R}^{m}, \mathbb{S}\right) \oplus u_{1} \mathcal{M}_{l_{1}-1}\left(\mathbb{R}^{m}, \mathbb{S}\right)
$$

The factor $u_{1}$ plays the role of embedding factor in this formula (Fischer decomposition).

Proposition 3 will be crucial in our reasoning, as it reveals a relation between solutions of HSD operators of different order, and a twistor operator. This will be the key ingredient to an inductive structure on the kernel of $\mathcal{Q}_{\lambda}$. We then conclude this section with the description of a special class of solutions for the operator $\mathcal{Q}_{\lambda}$, which are known as solutions of type A (see [9]). To do so, we need the following space of polynomials: 
Definition 6. For all integers $h \in \mathbb{N}$ and highest weights $\lambda^{\prime}=\left(l_{1}, \ldots, l_{k}\right)^{\prime}$, we define the vector space

$$
\mathcal{M}_{h ; \lambda}^{s}:=\left\{M_{h ; \lambda}\left(x ; u_{(k)}\right) \in \mathcal{P}_{h}\left(\mathbb{R}^{m}, \mathcal{S}_{\lambda}\right): \partial_{x} M_{h, \lambda}\left(x ; u_{(k)}\right)=0\right\} .
$$

This means that this vector space contains $\mathbb{S}$-valued polynomials which are monogenic in each of the variables $\left(x ; u_{(k)}\right)$ and satisfy extra conditions $\left\langle u_{i}, \partial_{j}\right\rangle M_{h ; \lambda}=0$ in the dummy variables (for $i<j$ ).

Remark 8. In the case where $k=1, \mathcal{M}_{h ; l_{1}}^{s}$ is the space of double monogenic polynomials, i.e. polynomials that are monogenic in both $x$ and $u_{1}$.

In view of the fact that the operator $\mathcal{Q}_{\lambda}$ can be seen as a suitable projection operator applied after the action of the operator $\partial_{x}$, see Definition 1 , it is clear that the vector space $\mathcal{M}_{h ; \lambda}^{s}$ contains $h$-homogeneous solutions for the operator $\mathcal{Q}_{\lambda}$. These are then precisely the solutions of type A. In [9], these spaces were decomposed into irreducible summands under the $\mathfrak{s o}(m)$-action, hereby relying heavily on the Howe dual pair $\operatorname{Spin}(m) \times \mathfrak{s p}(2 k)$ underlying Clifford analysis in $k$ vector variables. Note that this was done under the restriction that the degree in homogeneity satisfies $h \geq l_{1}$ :

Theorem 1. For all highest weights $\lambda^{\prime}=\left(l_{1}, \ldots, l_{k}\right)^{\prime}$ and integers $h \geq l_{1}$, one has that

$$
\mathcal{M}_{h ; \lambda}^{s} \cong \bigoplus_{\left(d_{1}, \ldots, d_{k}\right)} \mathcal{S}_{\mu(d)}
$$

where $\mu(d)$ denotes the dominant highest weight $\left(\mu_{0}, \mu_{1}, \ldots, \mu_{k}\right)^{\prime}$, with $\mu_{0}:=h+\sum_{j=1}^{k} d_{j}$ and $\mu_{j}=l_{j}-d_{j}$. In order for $\mu$ to be dominant, the integers $d_{j}$ must satisfy the conditions $0 \leq d_{j} \leq l_{j}-l_{j+1}$, for all $1 \leq j \leq k$.

It is now clear that if one knows how to relate arbitrary polynomial solutions to these special solutions of type A, that this automatically solves the question of how to decompose the former in terms of irreducible summands under the $\mathfrak{s o}(m)$-action. This approach will be worked out in the next section.

\section{$5 \quad$ Higher spin kernel decompositions}

From now on, we will fix an arbitrary highest weight $\lambda^{\prime}=\left(l_{1}, \ldots, l_{k}\right)^{\prime}$ with $k>2$ and focus on the following problem: How can one decompose the space

$$
\mathcal{K}_{h ; \lambda}:=\mathcal{P}_{h}\left(\mathbb{R}^{m}, \mathcal{S}_{\lambda}\right) \cap \operatorname{ker} \mathcal{Q}_{\lambda}
$$

as a (highly reducible) module under the regular action of the algebra $\mathfrak{s o}(m)$, with $h \in \mathbb{N}$ the degree of homogeneity of the polynomial solutions (i.e. in $x \in \mathbb{R}^{m}$ )?

Sometimes, it will be necessary to explicitly attach the highest weight as an index (i.e. in vector notation), but in that case we will omit the prime to enlighten the notations:

$$
\mathcal{K}_{h ; \lambda}=\mathcal{K}_{h ;\left(l_{1}, \ldots, l_{k}\right)} .
$$


Note that we exclude $k \in\{1,2\}$ as these polynomial kernel spaces have been described in respectively [5] and [2]. We briefly recall these results to illustrate the type of result we are after:

Theorem 2. For all integers $h \geq l_{1}>0$, the kernel of the Rarita-Schwinger operator $\mathcal{R}_{l_{1}}$ decomposes as follows:

$$
\begin{aligned}
\mathcal{K}_{h ;\left(l_{1}\right)} & :=\mathcal{P}_{h}\left(\mathbb{R}^{m}, \mathcal{M}_{l_{1}}\right) \cap \operatorname{ker} \mathcal{R}_{l_{1}} \\
& \cong\left(\mathcal{K}_{h ;\left(l_{1}\right)} \cap \operatorname{ker} \mathcal{T}^{(1)}\right) \oplus \bigoplus_{j_{1}=1}^{l_{1}}\left(\mathcal{K}_{h ;\left(l_{1}\right)} \cap \operatorname{ker}\left(\mathcal{T}^{(1)}\right)^{j_{1}+1}\right) /\left(\mathcal{K}_{h ;\left(l_{1}\right)} \cap \operatorname{ker}\left(\mathcal{T}^{(1)}\right)^{j_{1}}\right) \\
& \cong \bigoplus_{j_{1}=0}^{l_{1}} \mathcal{M}_{h-j_{1} ; l_{1}-j_{1}}^{s} \\
& =\bigoplus_{i_{1}=0}^{l_{1}} \mathcal{M}_{h-l_{1}+i_{1} ; i_{1}}^{s} .
\end{aligned}
$$

We can visualise the latter sum as a line of dots on an axis, whereby each dot represents the space $\mathcal{M}_{h-l_{1}+i_{1} ; i_{1}}^{s}$ (see Figure 1$)$.

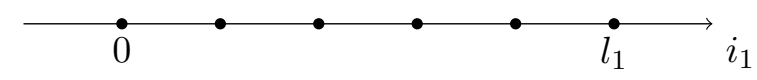

Figure 1: The kernel space of $\mathcal{R}_{l_{1}}$, where each dot represents a space $\mathcal{M}_{h-l_{1}+i_{1} ; i_{1}}^{s}$

Theorem 3. For all highest weights $\lambda^{\prime}=\left(l_{1}, l_{2}\right)^{\prime}$ and integers $h \geq l_{1}+l_{2}$, the kernel of the HSD operator $\mathcal{Q}_{\lambda}$ decomposes as follows:

$$
\begin{aligned}
& \mathcal{K}_{h ;\left(l_{1}, l_{2}\right)}:=\mathcal{P}_{h}\left(\mathbb{R}^{m}, \mathcal{S}_{\lambda}\right) \cap \operatorname{ker} \mathcal{Q}_{\lambda} \\
& \cong\left(\mathcal{K}_{h ;\left(l_{1}, l_{2}\right)} \cap \operatorname{ker} \mathcal{T}^{(2)}\right) \oplus \bigoplus_{j_{2}=1}^{l_{2}}\left(\mathcal{K}_{h ;\left(l_{1}, l_{2}\right)} \cap \operatorname{ker}\left(\mathcal{T}^{(2)}\right)^{j_{2}+1}\right) /\left(\mathcal{K}_{h ;\left(l_{1}, l_{2}\right)} \cap \operatorname{ker}\left(\mathcal{T}^{(2)}\right)^{j_{2}}\right) \\
& \cong \bigoplus_{j_{1}=0}^{l_{1}-l_{2}} \bigoplus_{j_{2}=0}^{l_{2}} \mathcal{M}_{h-j_{1}-j_{2} ; l_{1}-j_{1}, l_{2}-j_{2}}^{s} \\
& =\bigoplus_{i_{1}=l_{2}}^{l_{1}} \bigoplus_{i_{2}=0}^{l_{2}} \mathcal{M}_{h-l_{1}-l_{2}+i_{1}+i_{2} ; i_{1}, i_{2}}^{s} .
\end{aligned}
$$

The second sum is easier to interpret, as the summation indices $i_{p}$ represent the degree of homogeneity of $u_{p}$ of the polynomials in the spaces contained in the direct sum. We can visualise this sum over the dots in the rectangular grid in Figure 2.

By comparing Figure 1 and 2, we see a cuboid grid structure appearing in respectively a 1-dimensional and a 2-dimensional space. This emerging pattern raises the question whether this would be true in general, allowing us to formulate a proposition for general highest weights $\lambda^{\prime}$. 


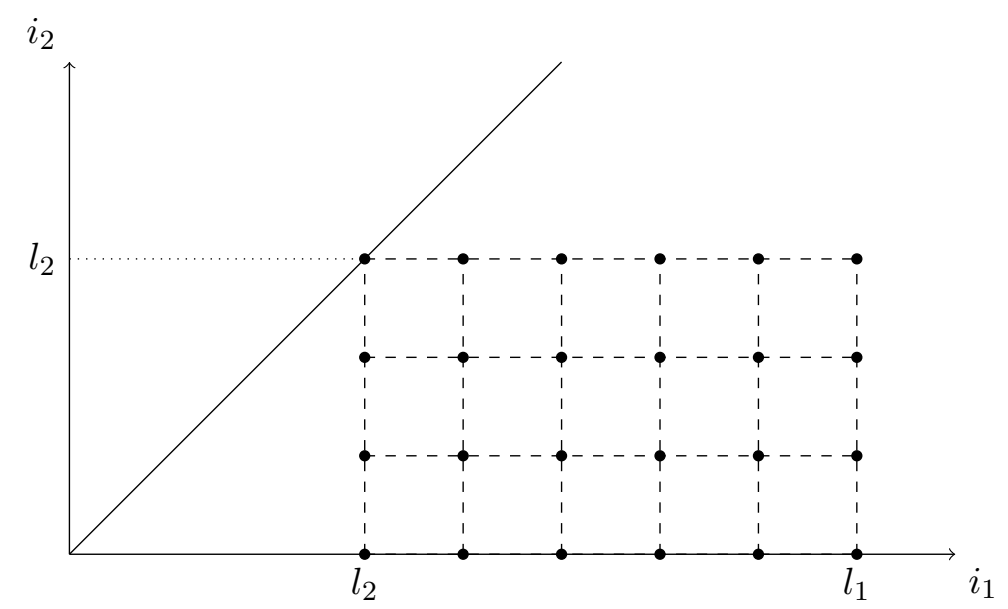

Figure 2: The kernel space of $\mathcal{Q}_{l_{1}, l_{2}}$, where each dot represents a space of type A solutions

Proposition 4. For all highest weights $\lambda^{\prime}=\left(l_{1}, \ldots, l_{k}\right)^{\prime}$ and integers $h \geq l_{1}+l_{2}$, the kernel of the HSD operator $\mathcal{Q}_{\lambda}$ decomposes as follows:

$$
\begin{aligned}
\mathcal{K}_{h ; \lambda} & :=\mathcal{P}_{h}\left(\mathbb{R}^{m}, \mathcal{S}_{\lambda}\right) \cap \operatorname{ker} \mathcal{Q}_{\lambda} \cong \bigoplus_{j_{1}=0}^{l_{1}-l_{2}} \cdots \bigoplus_{j_{k-1}=0}^{l_{k-1}-l_{k}} \bigoplus_{j_{k}=0}^{l_{k}} \mathcal{M}_{h-\sum_{p=1}^{k} j_{p} ; l_{1}-j_{1}, \ldots, l_{k}-j_{k}}^{s} \\
& =\mathcal{P}_{h}\left(\mathbb{R}^{m}, \mathcal{S}_{\lambda}\right) \cap \operatorname{ker} \mathcal{Q}_{\lambda} \cong \bigoplus_{i_{1}=l_{2}}^{l_{1}} \cdots \bigoplus_{i_{k-1}=l_{k}}^{l_{k}-1} \bigoplus_{i_{k}=0}^{l_{k}} \mathcal{M}_{h-\sum_{p=1}^{k}\left(l_{p}-i_{p}\right) ; i_{1}, \ldots, i_{k}}^{s} .
\end{aligned}
$$

Remark 9. The condition $h \geq l_{1}+l_{2}$ appearing in the proposition is easily explained. For each term in the direct sum to exist, we have to check the condition of Theorem 1, which translates here to $h-\sum_{p=1}^{k} j_{p} \geq l_{1}-j_{1}$ or $h \geq l_{1}+\sum_{p=2}^{k} j_{p}$. Due to the boundaries in the direct sum for $j_{p}(p \geq 2)$, the latter sum cannot exceed $l_{2}$.

In the remainder of this article, this is what we will investigate. Since the general case might be hard to grasp right away, we will illustrate our approach for the case $k=3$ first, and discuss $k>3$ afterwards.

\subsection{The case $k=3$}

We will prove that $\operatorname{ker}_{h} \mathcal{Q}_{l_{1}, l_{2}, l_{3}}$ has a cuboid grid structure, as predicted in (6), which is visualised in Figure 3. Throughout this subsection, we will assume $\lambda=\left(l_{1}, l_{2}, l_{3}\right)$, with $l_{1} \geq l_{2} \geq l_{3} \geq 0$, and $h \geq l_{1}+l_{2}$. We will define a grading onto this kernel space, hereby exploiting the different twistor operators. A first grading will be given by the twistor operator $\mathcal{T}^{(3)}$. Therefore, let us introduce the following spaces. 


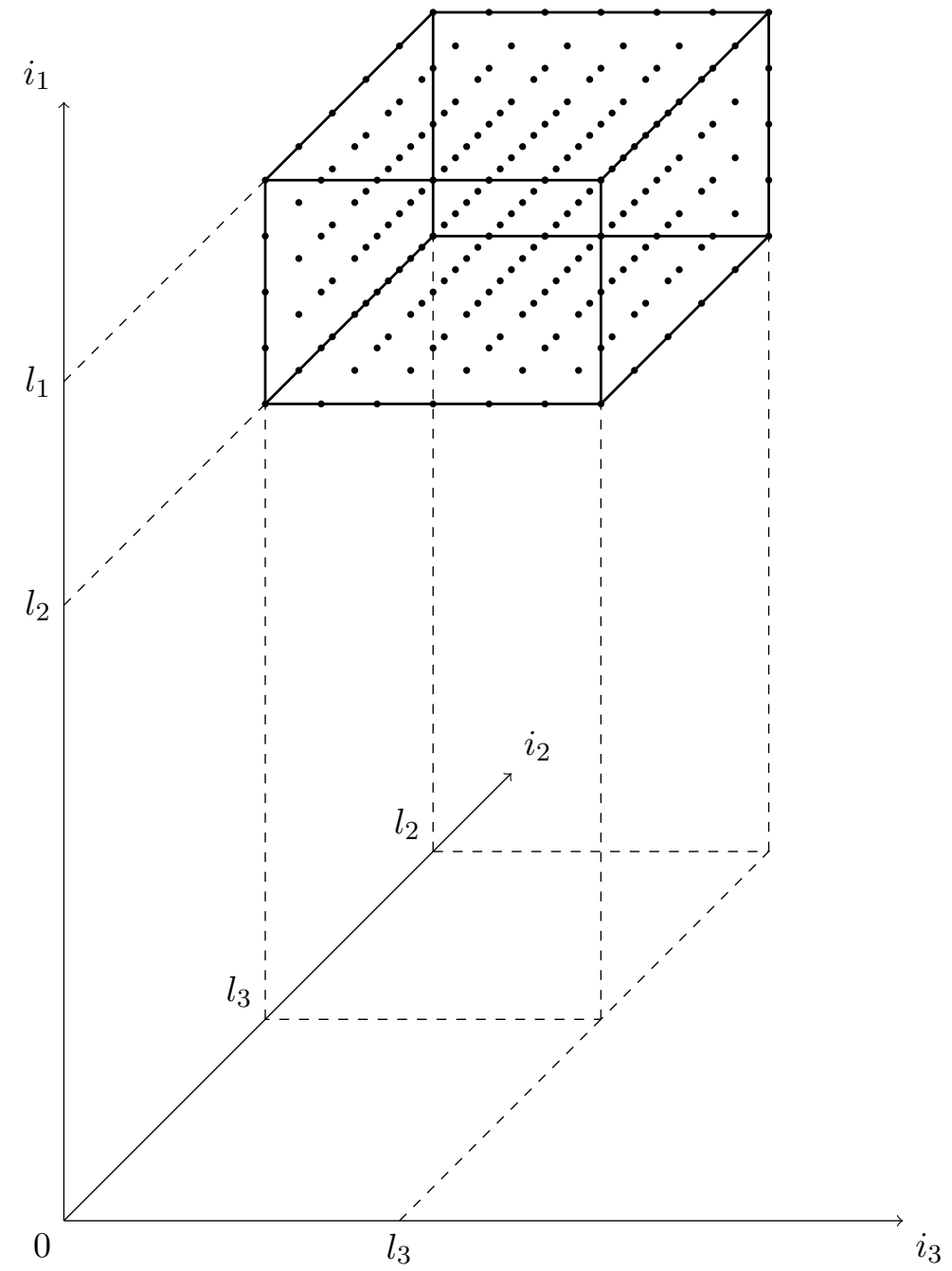

Figure 3: The kernel space of $\mathcal{Q}_{l_{1}, l_{2}, l_{3}}$, consisting of $\left(l_{1}-l_{2}+1\right)\left(l_{2}-l_{3}+1\right)\left(l_{3}+1\right)$ dots, where each dot represents a space of type A solutions

Definition 7. For arbitrary highest weights $\lambda^{\prime}=\left(l_{1}, l_{2}, l_{3}\right)^{\prime}$ with $l_{3}>0$, we put:

$$
\begin{aligned}
\mathcal{K}_{h ; \lambda}^{(0)} & :=\mathcal{K}_{h ; \lambda} \cap \operatorname{ker} \mathcal{T}^{(3)} \\
\mathcal{K}_{h ; \lambda}^{\left(j_{3}\right)} & :=\left(\mathcal{K}_{h ; \lambda} \cap \operatorname{ker}\left(\mathcal{T}^{(3)}\right)^{j_{3}+1}\right) /\left(\mathcal{K}_{h ; \lambda} \cap \operatorname{ker}\left(\mathcal{T}^{(3)}\right)^{j_{3}}\right) .
\end{aligned}
$$

We then have the following lemma

Lemma 4. For all $f \in \mathcal{C}^{\infty}\left(\mathbb{R}^{m}, \mathcal{S}_{\lambda}\right)$, we have that

$$
\left(\mathcal{T}^{(3)}\right)^{l_{3}+1} f=0 .
$$


Proof. Since $\operatorname{deg}_{u_{3}}(f)=l_{3}$, and $\mathcal{T}^{(3)}$ lowers the degree of $u_{3}$ by 1 , this is obviously true.

Remark 10. Keep in mind that a 'power' of a twistor operator is just a notation, as each consecutive twistor operator acts on a different space (since the degree of $u_{3}$ is lowered by one each time).

This lemma allows a decomposition of the form

$$
\mathcal{K}_{h ; \lambda} \cong \bigoplus_{j_{3}=0}^{l_{3}} \mathcal{K}_{h ; \lambda}^{\left(j_{3}\right)} .
$$

Assuming for now that we will indeed get a box structure, this decomposition can be visualised as in Figure 4, where the cuboid structure is split into $l_{3}+1$ rectangular slices, numbered from 0 to $l_{3}$, each slice representing a space $\mathcal{K}_{h ; \lambda}^{\left(j_{3}\right)}$ (with $0 \leq j_{3} \leq l_{3}$ ), counting from right to left.

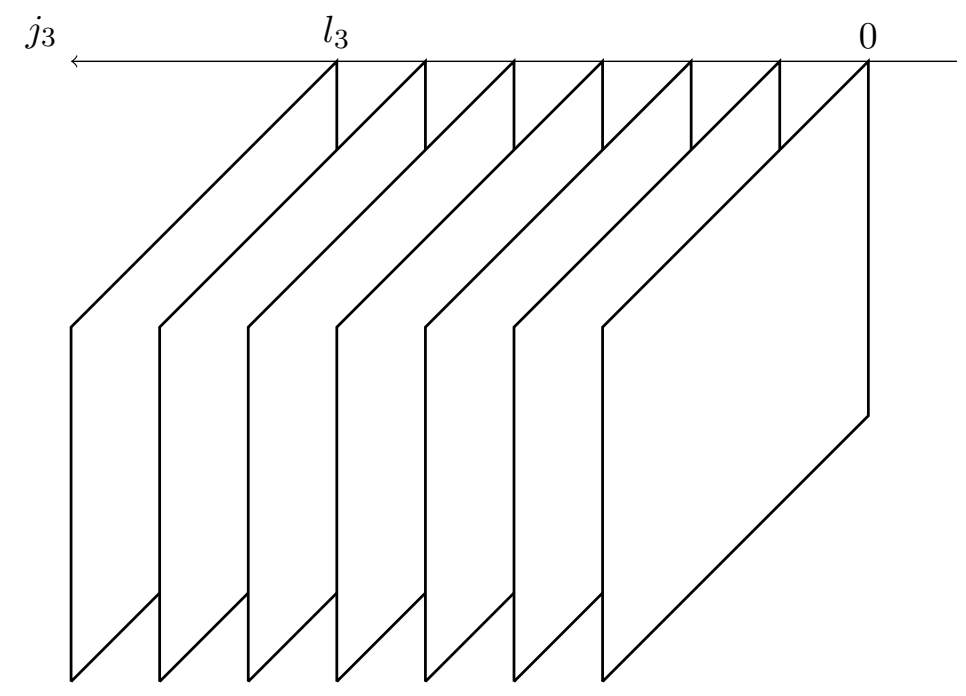

Figure 4: A first grading on $\mathcal{K}_{h ; l_{1}, l_{2}, l_{3}}$

We have now defined a grading on the kernel space of $\mathcal{Q}_{l_{1}, l_{2}, l_{3}}$ using the twistor operator $\mathcal{T}^{(3)}$. From Lemma 2, we know that twistor operators commute up to a Cartan factor. This means that we can define a second grading on the slices $\mathcal{K}_{h ; \lambda}^{\left(j_{3}\right)}$, this time using the twistor operator $\mathcal{T}^{(2)}$, which is independent of the first grading. Let us therefore introduce the following notations:

Definition 8. For arbitrary highest weights $\lambda^{\prime}=\left(l_{1}, l_{2}, l_{3}\right)^{\prime}$, we put:

$$
\begin{aligned}
\mathcal{K}_{h ; \lambda}^{\left(0, j_{3}\right)} & :=\mathcal{K}_{h ; \lambda}^{\left(j_{3}\right)} \cap \operatorname{ker} \mathcal{T}^{(2)} \\
\mathcal{K}_{h ; \lambda}^{\left(j_{2}, j_{3}\right)} & :=\left(\mathcal{K}_{h ; \lambda}^{\left(j_{3}\right)} \cap \operatorname{ker}\left(\mathcal{T}^{(2)}\right)^{j_{2}+1}\right) /\left(\mathcal{K}_{h ; \lambda}^{\left(j_{3}\right)} \cap \operatorname{ker}\left(\mathcal{T}^{(2)}\right)^{j_{2}}\right) .
\end{aligned}
$$


Remark 11. Note that we put the index $j_{2}$ before $j_{3}$, this is done according to the order of the twistor operators we use to define the gradings.

Similarly as above, we have the following lemma (the proof of which will be given in the next section, in full generality):

Lemma 5. For all $f \in \mathcal{C}^{\infty}\left(\mathbb{R}^{m}, \mathcal{S}_{\lambda}\right)$, we have that

$$
\left(\mathcal{T}^{(2)}\right)^{l_{2}-l_{3}+1} f=0 .
$$

We thus have a decomposition of the form

$$
\mathcal{K}_{h ; \lambda}^{\left(j_{3}\right)} \cong \bigoplus_{j_{2}=0}^{l_{2}-l_{3}} \mathcal{K}_{h ; \lambda}^{\left(j_{2}, j_{3}\right)} \Longrightarrow \mathcal{K}_{h ; \lambda} \cong \bigoplus_{j_{3}=0}^{l_{3}} \bigoplus_{j_{2}=0}^{l_{2}-l_{3}} \mathcal{K}_{h ; \lambda}^{\left(j_{2}, j_{3}\right)} .
$$

Graphically, the grading on the planes are represented by the dashed line segments in Figure 5. Each line segment stands for a space $\mathcal{K}_{h ; \lambda}^{\left(j_{2}, j_{3}\right)}$, where $j_{3}$ labels the rectangular slice, and $j_{2}$ labels the dashed line segments in the directions of the arrows. Until now, we

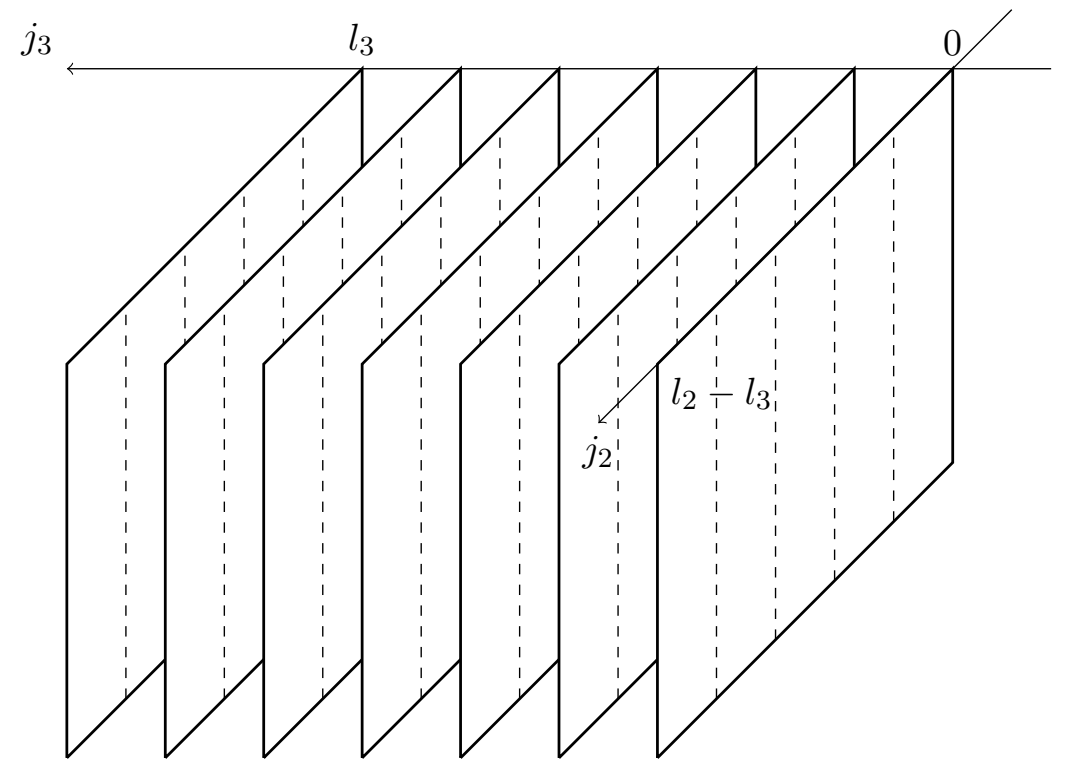

Figure 5: A second grading on $\mathcal{K}_{h ; l_{1}, l_{2}, l_{3}}$

have thus defined a grading using $\mathcal{T}^{(3)}$ and $\mathcal{T}^{(2)}$. We can define a third and final grading using the last twistor operator, $\mathcal{T}^{(1)}$, on the 'dashed line segments' $\mathcal{K}_{h ; \lambda}^{\left(j_{2}, j_{3}\right)}$. This gives rise to the following definition:

Definition 9. For arbitrary highest weights $\lambda^{\prime}=\left(l_{1}, l_{2}, l_{3}\right)^{\prime}$, we put:

$$
\begin{aligned}
\mathcal{K}_{h ; \lambda}^{\left(0, j_{2}, j_{3}\right)} & :=\mathcal{K}_{h ; \lambda}^{\left(j_{2}, j_{3}\right)} \cap \operatorname{ker} \mathcal{T}^{(1)} \\
\mathcal{K}_{h ; \lambda}^{\left(j_{1}, j_{2}, j_{3}\right)} & :=\left(\mathcal{K}_{h ; \lambda}^{\left(j_{2}, j_{3}\right)} \cap \operatorname{ker}\left(\mathcal{T}^{(1)}\right)^{j_{1}+1}\right) /\left(\mathcal{K}_{h ; \lambda}^{\left(j_{2}, j_{3}\right)} \cap \operatorname{ker}\left(\mathcal{T}^{(1)}\right)^{j_{1}}\right) .
\end{aligned}
$$


We then again have:

Lemma 6. For all $f \in \mathcal{C}^{\infty}\left(\mathbb{R}^{m}, \mathcal{S}_{\lambda}\right)$, we have that

$$
\left(\mathcal{T}^{(1)}\right)^{l_{1}-l_{2}+1} f=0 .
$$

This thus leads to

$$
\mathcal{K}_{h ; \lambda}^{\left(j_{2}, j_{3}\right)} \cong \bigoplus_{j_{1}=0}^{l_{1}-l_{2}} \mathcal{K}_{h ; \lambda}^{\left(j_{1}, j_{2}, j_{3}\right)} \Longrightarrow \mathcal{K}_{h ; \lambda} \cong \bigoplus_{j_{3}=0}^{l_{3}} \bigoplus_{j_{2}=0}^{l_{2}-l_{3} l_{1}-l_{2}} \bigoplus_{j_{1}=0}^{\mathcal{K}_{h ; \lambda}^{\left(j_{1}, j_{2}, j_{3}\right)}}
$$

Graphically, the final grading on the dashed line segments is depicted by means of the dots in Figure 6. Each dot here represents a space $\mathcal{K}_{h ; \lambda}^{\left(j_{1}, j_{2}, j_{3}\right)}$.

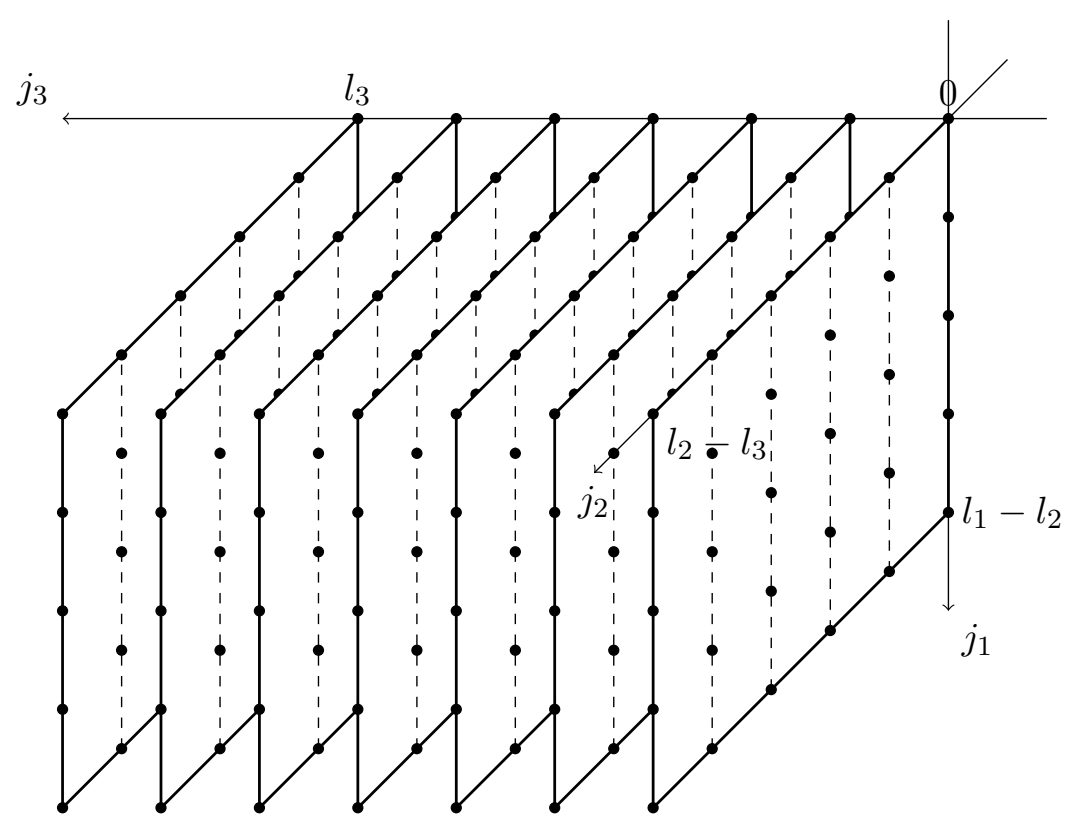

Figure 6: A third and final grading on $\mathcal{K}_{h ; l_{1}, l_{2}, l_{3}}$

Remark 12. The problem at hand, i.e. describing the kernel space of $\mathcal{Q}_{l_{1}, l_{2}, l_{3}}$, is now reduced to describing the spaces $\mathcal{K}_{h ; \lambda}^{\left(j_{1}, j_{2}, j_{3}\right)}$.

\subsection{The general case}

Let us now take a look at the general case. From now on, we will assume $\lambda=\left(l_{1}, \ldots, l_{k}\right)$ with $l_{1} \geq \cdots \geq l_{k}>0$ and In order to decompose $\mathcal{K}_{h ; \lambda}$ for arbitrary half-integer highest weights, we first of all define a grading on the kernel space using the twistor operator $\mathcal{T}^{(k)}$, inspired by Definition 7: 
Definition 10. For arbitrary highest weights $\lambda^{\prime}=\left(l_{1}, \ldots, l_{k}\right)^{\prime}$ with $l_{k}>0$, we put:

$$
\begin{aligned}
\mathcal{K}_{h ; \lambda}^{(0)} & :=\mathcal{K}_{h ; \lambda} \cap \operatorname{ker} \mathcal{T}^{(k)} \\
\mathcal{K}_{h ; \lambda}^{\left(j_{k}\right)} & :=\left(\mathcal{K}_{h ; \lambda} \cap \operatorname{ker}\left(\mathcal{T}^{(k)}\right)^{j_{k}+1}\right) /\left(\mathcal{K}_{h ; \lambda} \cap \operatorname{ker}\left(\mathcal{T}^{(k)}\right)^{j_{k}}\right) .
\end{aligned}
$$

The following lemma will then again lead to a direct sum decomposition.

Lemma 7. For all $f \in \mathcal{C}^{\infty}\left(\mathbb{R}^{m}, \mathcal{S}_{\lambda}\right)$, we have that

$$
\left(\mathcal{T}^{(k)}\right)^{l_{k}+1} f=0
$$

Proof. Since $\operatorname{deg}_{u_{k}}(f)=l_{k}$, and $\mathcal{T}^{(k)}$ lowers the degree in $u_{k}$ by 1 , this is trivial.

From Lemma 7, and Definition 10, we thus have:

$$
\mathcal{K}_{h ; \lambda} \cong \bigoplus_{j_{k}=0}^{l_{k}} \mathcal{K}_{h ; \lambda}^{\left(j_{k}\right)}
$$

Remark 13. Comparing this to the case $k=3$, where this translated into a decomposition into rectangular slices, this amounts to a decomposition in rectangular hypercuboid slices.

Since $\left\langle\partial_{x}, \partial_{k}\right\rangle=\mathcal{T}_{\lambda}^{(k)}$ when acting on $\mathcal{S}_{\lambda}$-valued polynomials, one immediately sees that

$$
\mathcal{K}_{h ; \lambda}^{(0)}=\mathcal{P}_{h}\left(\mathbb{R}^{m}, \mathcal{S}_{\lambda}\right) \cap \operatorname{ker} \mathcal{Q}_{l_{1}, \ldots, l_{k-1}}^{T} .
$$

In view of the fact that the twisted operator is essentially the HSD operator $\mathcal{Q}_{l_{1}, \ldots, l_{k-1}}$ acting on $\mathcal{S}_{\lambda}$-valued functions, this clearly suggests using induction on the length of the highest weight of the underlying representation characterising the values.

Lemma 8. Given an arbitrary highest weight $\lambda^{\prime}=\left(l_{1}, \ldots, l_{k}\right)^{\prime}$ with $l_{k}>0$, one has:

$$
\left\langle\partial_{x}, \partial_{k}\right\rangle \mathcal{Q}_{\lambda}=\frac{m+2 \mathbb{E}_{k}-2 k}{m+2 \mathbb{E}_{k}-2 k+2} \mathcal{Q}_{\lambda-L_{k}}\left\langle\partial_{x}, \partial_{k}\right\rangle
$$

Proof. This follows directly from the fact that $\left\langle\partial_{x}, \partial_{k}\right\rangle=\mathcal{T}_{\lambda}^{(k)}$, and Lemma 2.

This lemma actually tells us that for each $f \in \operatorname{ker} \mathcal{Q}_{\lambda}$, we have that either $\left\langle\partial_{x}, \partial_{k}\right\rangle f=0$, or $\left\langle\partial_{x}, \partial_{k}\right\rangle f \in \operatorname{ker} \mathcal{Q}_{\lambda-L_{k}}$. Or more generally, we have the following theorem.

Theorem 4. Given a fixed highest weight $\lambda^{\prime}=\left(l_{1}, \ldots, l_{k}\right)^{\prime}$ and an integer $h \in \mathbb{N}$, one has the following property for all $1 \leq j_{k} \leq l_{k}$ :

$$
\varphi_{k ; j_{k}}:=\left\langle\partial_{x}, \partial_{k}\right\rangle^{j_{k}}: \mathcal{K}_{h ; \lambda}^{\left(j_{k}\right)} \rightarrow \mathcal{K}_{h-j_{k} ; \lambda-j_{k} L_{k}}^{(0)} .
$$

Proof. From Definition 10, it follows that the operator $\left\langle\partial_{x}, \partial_{k}\right\rangle^{j_{k}}$ maps polynomials in $\mathcal{K}_{h ; \lambda}^{\left(j_{k}\right)}$ to elements of the vector space $\operatorname{ker}\left\langle\partial_{x}, \partial_{k}\right\rangle$. Together with Lemma 8, this then proves the assertion. 
Remark 14. Note that the target space at the right-hand side of (8) contains solutions for the twisted version of the operator $\mathcal{Q}_{l_{1}, \ldots, l_{k-1}}$, acting on $\mathcal{S}_{\lambda-j_{k} L_{k}}$-valued functions.

Remark 15. Note that $\left\langle\partial_{x}, \partial_{k}\right\rangle$ is the twistor operator $\mathcal{T}^{(k)}$, which results in the fact that $\varphi_{k ; j_{k}}=\left(\mathcal{T}^{(k)}\right)^{j_{k}}$, keeping in mind that we can 'write' a power of a twistor operator due to the fact that we use the Euler notations. One should realise that each consecutive twistor operator actually acts on a different polynomial space, as the degree of $u_{k}$ lowers with each consecutive action of $\mathcal{T}^{(k)}$.

Remark 16. It is hereby crucial to point out that the mappings $\varphi_{k ; j_{k}}$ from the previous proposition are not necessarily surjective, which means that not all irreducible summands in the 0-graded image space at the right-hand side (containing null solutions for a twisted HSD operator) will be present in the $j_{k}$-graded subspace at the left-hand side of the arrow.

From Theorem 4, the question arises whether an analogue of this theorem holds for the other twistor operators as well. This is indeed the case, as we will show in the following theorem, but first we need a lemma.

Lemma 9. For all $f \in \mathcal{C}^{\infty}\left(\mathbb{R}^{m}, \mathcal{S}_{\lambda}\right)$, we have that

$$
\left(\mathcal{T}^{(a)}\right)^{l_{a}-l_{a+1}+1} f=0 .
$$

Proof. Denoting $g=\left(\mathcal{T}^{(a)}\right)^{l_{a}-l_{a+1}} f$, we have that $g \in \mathcal{C}^{\infty}\left(\mathbb{R}^{m}, \mathcal{S}_{l_{1}, \ldots, l_{a-1}, l_{a+1}, l_{a+1}, \ldots, l_{k}}\right)$ where $\operatorname{deg}_{u_{a}}(g)=\operatorname{deg}_{u_{a+1}}(g)=l_{a+1}$. Remember that $\mathcal{S}_{\lambda}$ is generated by (1). Moreover, the highest weight vector of $\mathcal{S}_{l_{1}, \ldots, l_{a-1}, l_{a+1}, l_{a+1}, \ldots, l_{k}}$ is symmetrical in $u_{a}$ and $u_{a+1}$. Since $\left\langle u_{a+1}, \partial_{a}\right\rangle$ is $\operatorname{Spin}(m)$-invariant, this means that

$$
\left\langle u_{a+1}, \partial_{a}\right\rangle g=0
$$

or $g$ is an element in the kernel of an extra operator which is not in the definition of simplicial monogenicity. We find that

$$
\mathcal{T}^{(a)} g=\prod_{j=a+1}^{k}\left(1-\frac{\left\langle u_{j}, \partial_{a}\right\rangle\left\langle u_{a}, \partial_{j}\right\rangle}{\mathbb{E}_{a}-\mathbb{E}_{j}+j-a+1}\right)\left\langle\partial_{a}, \partial_{x}\right\rangle g .
$$

Using the relation $\left\langle u_{a+1}, \partial_{a}\right\rangle\left\langle u_{a}, \partial_{a+1}\right\rangle=\left\langle u_{a}, \partial_{a+1}\right\rangle\left\langle u_{a+1}, \partial_{a}\right\rangle+\mathbb{E}_{a+1}-\mathbb{E}_{a}$, this equals

$$
\left\langle u_{a}, \partial_{a+1}\right\rangle\left\langle u_{a+1}, \partial_{a}\right\rangle \prod_{j=a+2}^{k}\left(1-\frac{\left\langle u_{j}, \partial_{a}\right\rangle\left\langle u_{a}, \partial_{j}\right\rangle}{\mathbb{E}_{a}-\mathbb{E}_{j}+j-a+1}\right)\left\langle\partial_{a}, \partial_{x}\right\rangle g .
$$

Inductively running $\left\langle u_{a+1}, \partial_{a}\right\rangle$ through each factor, using the fact that $\left\langle u_{a+1}, \partial_{j}\right\rangle g=0$, for all $j>a+1$, and (9), we find that

$$
\mathcal{T}^{(a)} g=0
$$

This proves the lemma. 
This lemma is crucial for what follows, and basically gives us an upper boundary on the number of times a twistor operator on $\mathcal{K}_{h, \lambda}$ until the result becomes trivial. Also, let us recall a result that was proved in [8]. It essentially tells us that certain twistor operator compositions are trivial. For the sake of completeness we briefly recall the proof here.

Proposition 5. For a fixed highest weight $\lambda=\left(l_{1}, \ldots, l_{j-2}, l_{j}, l_{j}, l_{j+1}, \ldots, l_{k}\right)^{\prime}$ (note that $\left.l_{j}=l_{j-1}\right)$, one has that

$$
\mathcal{T}_{l_{1}, \ldots, l_{j-2}, l_{j}, l_{j}-1, l_{j+1}, \ldots, l_{k}}^{(j-1)} \circ \mathcal{T}_{l_{1}, \ldots, l_{j-2}, l_{j}, l_{j}, l_{j+1}, \ldots, l_{k}}^{(j)} \equiv 0 .
$$

Proof. First of all, we note that this twistor operator composition connects the following three dominant highest weights:

$$
\begin{aligned}
\left(l_{1}, \ldots, l_{j-2}, l_{j}, l_{j}, l_{j+1}, \ldots, l_{k}\right)^{\prime}= & \left(l_{1}-1, \ldots, l_{j-2}-1, l_{j}-1, l_{j}-1, l_{j+1}, \ldots, l_{k}\right)^{\prime} \\
& +(1, \ldots, 1,1,1,0, \ldots, 0) \\
\left(l_{1}, \ldots, l_{j-2}, l_{j}, l_{j}-1, l_{j+1}, \ldots, l_{k}\right)^{\prime}= & \left(l_{1}-1, \ldots, l_{j-2}-1, l_{j}-1, l_{j}-1, l_{j+1}, \ldots, l_{k}\right)^{\prime} \\
& +(1, \ldots, 1,1,0,0, \ldots, 0) \\
\left(l_{1}, \ldots, l_{j-2}, l_{j}-1, l_{j}-1, l_{j+1}, \ldots, l_{k}\right)^{\prime}= & \left(l_{1}-1, \ldots, l_{j-2}-1, l_{j}-1, l_{j}-1, l_{j+1}, \ldots, l_{k}\right)^{\prime} \\
& +(1, \ldots, 1,0,0,0, \ldots, 0) .
\end{aligned}
$$

In other words, the highest weights in the composition all sit inside the tensor product of one fixed highest weight (call it $\mu$ ) and three fundamental highest weights, which we will denote by means of $\omega_{+}, \omega_{0}$ and $\omega_{-}$respectively (in that order). As a matter of fact, the spaces $\mathcal{S}_{\lambda}$ associated to the dominant weights at the left-hand side are precisely the Cartan products of the fixed space $\mathcal{S}_{\mu}$ and the representations $\mathbb{V}_{\omega}^{ \pm}$and $\mathbb{V}_{\omega}^{0}$. The latter correspond to exterior powers of the fundamental representation, and this means that the sequence of differential operators $\mathbb{V}_{\omega}^{+} \rightarrow \mathbb{V}_{\omega}^{0} \rightarrow \mathbb{V}_{\omega}^{-}$is nothing but a part of the de Rham sequence for the codifferential operator. If we twist this particular sequence with the representation $\mathcal{S}_{\mu}$ (i.e. considering $\mathcal{S}_{\mu}$-valued forms), we still get a composition which is trivial. So is the restriction and projection of this composition to the Cartan products. Now, in view of the fact that the only first-order operators acting between the Cartan products are the aforementioned twistor operators, we have that the composition

$$
\mathcal{T}_{l_{1}, \ldots, l_{j-2}, l_{j}, l_{j}-1, l_{j+1}, \ldots, l_{k}}^{(j-1)} \circ \mathcal{T}_{\left(l_{1}, \ldots, l_{j-2}, l_{j}, l_{j}, l_{j+1}, \ldots, l_{k}\right)}^{(j)}
$$

is indeed zero.

Let us first focus on the space $\mathcal{K}_{h ; \lambda}^{(0)}$, which would be the first hyperslice (compare to Figure 4). Inspired by Definition 10, we define the following spaces:

Definition 11. For arbitrary highest weights $\lambda^{\prime}=\left(l_{1}, \ldots, l_{k}\right)^{\prime}$, we put:

$$
\begin{aligned}
\mathcal{K}_{h ; \lambda}^{\left(0, j_{i+1}, \ldots, j_{k}\right)} & :=\mathcal{K}_{h ; \lambda}^{\left(j_{i+1}, \ldots, j_{k}\right)} \cap \operatorname{ker} \mathcal{T}^{(i)} \\
\mathcal{K}_{h ; \lambda}^{\left(j_{i}, j_{i+1}, \ldots, j_{k}\right)} & :=\left(\mathcal{K}_{h ; \lambda}^{\left(j_{i+1}, \ldots, j_{k}\right)} \cap \operatorname{ker}\left(\mathcal{T}^{(i)}\right)^{j_{i}+1}\right) /\left(\mathcal{K}_{h ; \lambda}^{\left(j_{i+1}, \ldots, j_{k}\right)} \cap \operatorname{ker}\left(\mathcal{T}^{(i)}\right)^{j_{i}}\right) .
\end{aligned}
$$


Remark 17. It is instructive to realise that the number of upper indices is actually the codimension of the graphical interpretation of the space. For the case $k=3$, we have for instance that $\mathcal{K}_{h ; \lambda}^{\left(j_{3}\right)}$ is a rectangular slice of codimension $1, \mathcal{K}_{h ; \lambda}^{\left(j_{2}, j_{3}\right)}$ is a line segment of codimension 2, and $\mathcal{K}_{h ; \lambda}^{\left(j_{1}, j_{2}, j_{3}\right)}$ is a dot of codimension 3 .

With this definition, we can state the following proposition.

Proposition 6. The kernel space of $\mathcal{Q}_{\lambda}$ has the following decomposition:

$$
\mathcal{K}_{h ; \lambda} \cong \bigoplus_{j_{1}=0}^{l_{1}-l_{2}} \cdots \bigoplus_{j_{k-1}=0}^{l_{k-1}-l_{k}} \bigoplus_{j_{k}=0}^{l_{k}} \mathcal{K}_{h ; \lambda}^{\left(j_{1}, j_{2}, \ldots, j_{k}\right)}
$$

Proof. Using Lemma 9 and Proposition 5 we have for all $0 \leq j_{k} \leq l_{k}$ that

$$
\mathcal{K}_{h ; \lambda}^{\left(j_{k}\right)} \cong \bigoplus_{j_{k-1}=0}^{l_{k-1}-l_{k}} \mathcal{K}_{h ; \lambda}^{\left(j_{k-1}, j_{k}\right)}
$$

and in general, that

$$
\mathcal{K}_{h ; \lambda}^{\left(j_{i+1}, \ldots, j_{k}\right)} \cong \bigoplus_{j_{i}=0}^{l_{i}-l_{i+1}} \mathcal{K}_{h ; \lambda}^{\left(j_{i}, j_{i+1}, \ldots, j_{k}\right)} .
$$

Using this argument inductively on $\mathcal{K}_{h ; \lambda}$ we get a full decomposition of the 'hyperrectangle' in 'dots'.

\subsection{Interpreting the spaces $\mathcal{K}_{h ; \lambda}^{\left(j_{1}, j_{2}, \ldots, j_{k}\right)}$}

First of all, we will take a closer look the component $\mathcal{K}_{h ; \lambda}^{(0, \ldots, 0)}$ of this direct sum.

Lemma 10. We have the following equality:

$$
\mathcal{K}_{h ; \lambda}^{(0, \ldots, 0)}=\mathcal{K}_{h ; \lambda} \cap \operatorname{ker}\left\{\left\langle\partial_{x}, \partial_{i}\right\rangle: i \in\{1, \ldots, k\}\right\} .
$$

Proof. From the definition of $\mathcal{K}_{h ; \lambda}^{(0, \ldots, 0)}$, we know that any $f \in \mathcal{K}_{h ; \lambda}^{(0, \ldots, 0)}$ satisfies the relation $\mathcal{T}^{(i)} f=0$, for all $1 \leq i \leq k$. The $k$-th twistor operator is defined as

$$
\mathcal{T}^{(k)}=\left\langle\partial_{k}, \partial_{x}\right\rangle
$$

thus $\left\langle\partial_{k}, \partial_{x}\right\rangle f=0$. It then follows that

$$
\begin{array}{r}
0=\mathcal{T}^{(k-1)} f=\left(1+\frac{1}{\mathbb{E}_{k}-\mathbb{E}_{k-1}+k-1-(k+1)}\left\langle u_{k}, \partial_{k-1}\right\rangle\left\langle u_{k-1}, \partial_{k}\right\rangle\right) \\
=\left\langle\partial_{k-1}, \partial_{x}\right\rangle f \\
=\left\langle\partial_{k-1}, \partial_{x}\right\rangle f,
\end{array}
$$


where we have used that $\left[\left\langle u_{k-1}, \partial_{k}\right\rangle,\left\langle\partial_{k-1}, \partial_{x}\right\rangle\right]=-\left\langle\partial_{k}, \partial_{x}\right\rangle$. Continuing this argument inductively on the twistor operators, we find that

$$
0=\mathcal{T}^{(j)} f=\prod_{p=j+1}^{k}\left(1+\frac{1}{\mathbb{E}_{p}-\mathbb{E}_{j}+j-(p+1)}\left\langle u_{p}, \partial_{j}\right\rangle\left\langle u_{j}, \partial_{p}\right\rangle\right)\left\langle\partial_{j}, \partial_{x}\right\rangle f=\left\langle\partial_{j}, \partial_{x}\right\rangle f
$$

This proves that

$$
\mathcal{K}_{h ; \lambda}^{(0, \ldots, 0)} \subseteq \mathcal{K}_{h ; \lambda} \cap \operatorname{ker}\left\{\left\langle\partial_{x}, \partial_{i}\right\rangle: i \in\{1, \ldots, k\}\right\} .
$$

From the fact that each twistor operator can be written as (3), whereby each term ends with an operator of the form $\left\langle\partial_{i}, \partial_{x}\right\rangle$, follows the inverse inclusion, finishing the proof.

We can then link this space to the type A solutions defined in section 4 .

Theorem 5. One has that $\mathcal{K}_{h ; \lambda}^{(0, \ldots, 0)}=\mathcal{M}_{h ; \lambda}^{s}$.

Proof. We have that

$$
\mathcal{K}_{h ; \lambda}^{(0, \ldots, 0)}=\mathcal{K}_{h ; \lambda} \cap \operatorname{ker}\left\{\left\langle\partial_{x}, \partial_{i}\right\rangle: i \in\{1, \ldots, k\}\right\},
$$

so for all $f \in \mathcal{K}_{h ; \lambda}^{(0, \ldots, 0)} \subset \mathcal{P}_{h}\left(\mathbb{R}^{m}, \mathcal{S}_{\lambda}\right)$, we have

$$
0=\mathcal{Q}_{\lambda} f=\prod_{j=1}^{k}\left(1+\frac{u_{j} \partial_{j}}{2 \mathbb{E}_{j}+m-2}\right) \partial_{x}=\partial_{x} f
$$

since $\partial_{i} \partial_{x}=-\partial_{x} \partial_{i}-2\left\langle\partial_{i}, \partial_{x}\right\rangle$. Thus we have proved that

$$
\mathcal{K}_{h ; \lambda}^{(0, \ldots, 0)} \subseteq \mathcal{M}_{h ; \lambda}^{s}
$$

On the other hand, for all $g \in \mathcal{M}_{h ; \lambda}^{s}$, we have that

$$
\left\langle\partial_{i}, \partial_{x}\right\rangle g=\frac{1}{2}\left(\partial_{i} \partial_{x}+\partial_{x} \partial_{i}\right) g=0,
$$

thus

$$
\mathcal{M}_{h ; \lambda}^{s} \subseteq \mathcal{K}_{h ; \lambda} \cap \operatorname{ker}\left\{\left\langle\partial_{x}, \partial_{i}\right\rangle: i \in\{1, \ldots, k\}\right\}=\mathcal{K}_{h ; \lambda}^{(0, \ldots, 0)},
$$

the inverse inclusion, which finishes the proof.

Thus far, we have been able to describe the space $\mathcal{K}_{h ; \lambda}^{(0, \ldots, 0)}$. Let us take a look at the other components of the decomposition (10). Let us introduce some operators through the following theorem.

Theorem 6. Given a fixed highest weight $\lambda^{\prime}=\left(l_{1}, \ldots, l_{k}\right)^{\prime}$ and an integer $h \in \mathbb{N}$, one has the following property for all $1 \leq i \leq k-1$ and all $1 \leq j_{i} \leq l_{i}-l_{i+1}$ :

$$
\begin{aligned}
\varphi_{i ; j_{i}}:=\left(\mathcal{T}^{(i)}\right)^{j_{i}}: & \\
& \left(\mathcal{K}_{h ; \lambda} \cap \operatorname{ker}\left(\mathcal{T}^{(i)}\right)^{j_{i}+1}\right) /\left(\mathcal{K}_{h ; \lambda} \cap \operatorname{ker}\left(\mathcal{T}^{(i)}\right)^{j_{i}}\right) \rightarrow \mathcal{K}_{h-j_{i} ; \lambda-j_{i} L_{i}} \cap \operatorname{ker} \mathcal{T}^{(i)} .
\end{aligned}
$$


Remark 18. The operators $\varphi_{i ; j_{i}}$ give us a way to relate functions in $\operatorname{ker} \mathcal{Q}_{\lambda} \cap \operatorname{ker}\left(\mathcal{T}^{(i)}\right)^{j_{i}}$ to functions in the kernel of another HSD operator and the kernel of $\mathcal{T}^{(i)}$ itself. In a sense, this operator gives us a way to 'lower' the upper index $j_{i}$ in Definition 11.

Proof. This follows directly from Lemma 3. Lemma 9 gives us a lower boundary for $j$ for the space $\left(\mathcal{K}_{h ; \lambda} \cap \operatorname{ker}\left(\mathcal{T}^{(i)}\right)^{j+1}\right)$ to become trivial.

From the definition of $\mathcal{K}_{h ; \lambda}^{\left(j_{1}, j_{2}, \ldots, j_{k}\right)}$, and the properties of the operators $\varphi_{i ; j_{i}}$, we find that

$$
\begin{aligned}
\mathcal{K}_{h ; \lambda}^{\left(j_{1}, j_{2}, \ldots, j_{k}\right)} & \cong\left(\varphi_{1, j_{1}} \cdots \varphi_{k-1, j_{k-1}} \varphi_{k, j_{k}} \mathcal{K}_{h, \lambda}\right) \cap \operatorname{ker}\left(\mathcal{T}^{(1)}, \mathcal{T}^{(2)}, \ldots, \mathcal{T}^{(k)}\right) \\
& =\left(\left(\mathcal{T}^{(k)}\right)^{j_{k}}\left(\mathcal{T}^{(k-1)}\right)^{j_{k-1}} \cdots\left(\mathcal{T}^{(1)}\right)^{j_{1}} \mathcal{K}_{h, \lambda}\right) \cap \operatorname{ker}\left(\mathcal{T}^{(1)}, \mathcal{T}^{(2)}, \ldots, \mathcal{T}^{(k)}\right) .
\end{aligned}
$$

Using the same argument from Lemma 10, we get

$$
\mathcal{K}_{h ; \lambda}^{\left(j_{1}, j_{2}, \ldots, j_{k}\right)} \cong\left(\left\langle\partial_{1}, \partial_{x}\right\rangle^{j_{1}} \ldots\left\langle\partial_{k}, \partial_{x}\right\rangle^{j_{k}} \mathcal{K}_{h, \lambda}\right) \cap \operatorname{ker}\left(\left\langle\partial_{1}, \partial_{x}\right\rangle, \ldots,\left\langle\partial_{k}, \partial_{x}\right\rangle\right) .
$$

Then the properties of the operators $\varphi_{i ; j_{i}}$ tell us that

$$
\mathcal{K}_{h ; \lambda}^{\left(j_{1}, j_{2}, \ldots, j_{k}\right)} \subseteq \mathcal{K}_{h-\sum_{i=1}^{k} j_{i}, l_{1}-j_{1}, \ldots, l_{k}-j_{k}}^{(0, \ldots, 0)} .
$$

Using Theorem 5, we find that

$$
\mathcal{K}_{h ; \lambda}^{\left(j_{1}, j_{2}, \ldots, j_{k}\right)} \subseteq \mathcal{M}_{h-\sum_{i=1}^{k} j_{i}, l_{1}-j_{1}, \ldots, l_{k}-j_{k}}^{s} .
$$

We know how a space $\mathcal{M}_{h, \lambda}^{s}$ decomposes (see Theorem 1). This means that if we can prove the inverse inclusion of (11), we have found a full decomposition of $\mathcal{K}_{h ; \lambda}$. In order to try and prove this, we will attempt to count the dimensions of both spaces. Therefore, we need a generalised CK-extension.

\subsection{A generalised CK-extension}

In the classical case, there exists a CK-extension related to the Dirac operator (e.g. [11]), which allows the construction of monogenic functions (null solutions of the Dirac operator) from functions in one variable less. In this subsection, we try to find out if a similar method can be found to construct solutions of higher spin Dirac operators. Remember that the higher spin Dirac operator is defined as follows

$$
\mathcal{Q}_{\lambda}=p_{\mathfrak{o s p}(1,2 k)} \partial_{x}=\prod_{j=1}^{k}\left(1+\frac{u_{j} \partial_{j}}{m+2 \mathbb{E}_{i}-2 i}\right) \partial_{x}: \mathcal{C}^{\infty}\left(\mathbb{R}^{m}, \mathcal{S}_{\lambda}\right) \rightarrow \mathcal{C}^{\infty}\left(\mathbb{R}^{m}, \mathcal{S}_{\lambda}\right) .
$$

We define a vector variable and the Dirac operator in $\mathbb{R}^{m-1}$ as

$$
x^{*}=\sum_{j=1}^{m-1} e_{j} x_{j} \text { and } \partial_{x^{*}}=\sum_{j=1}^{m-1} e_{j} \partial_{x_{j}} .
$$


Then we can also define the higher spin Dirac operator on $\mathbb{R}^{m-1}$ as

$$
\mathcal{Q}_{\lambda}^{*}=p_{\mathfrak{o s p}(1,2 k)} \partial_{x^{*}}
$$

For any function $f$ one has that

$$
f \in \operatorname{ker}_{h} \mathcal{Q}_{\lambda} \Leftrightarrow \partial_{x_{m}} f=-\left(p_{\mathfrak{o s p}(1,2 k)} e_{m}\right)^{-1} \mathcal{Q}_{\lambda}^{*} f .
$$

Obviously, $p_{\mathfrak{o s p}(1,2 k)} e_{m}$ needs to be invertible (see next lemma). The unique solution to this first order differential equation is given by

$$
\begin{aligned}
f(x) & =e^{-x_{m}\left(p_{\mathfrak{o s p}(1,2 k)} e_{m}\right)^{-1} \mathcal{Q}_{\lambda}^{*}} f\left(x^{*}, 0\right) \\
& =\sum_{j=0}^{\infty} \frac{\left(-x_{m}\right)^{j}}{j !}\left(\left(p_{\mathfrak{o s p}(1,2 k)} e_{m}\right)^{-1} \mathcal{Q}_{\lambda}^{*}\right)^{j} f\left(x^{*}, 0\right) .
\end{aligned}
$$

Lemma 11. The operator $p_{\mathfrak{o s p}(1,2 k)} e_{m}$ is invertible.

Proof. From [8, Theorem 6], we have that

$$
\Delta^{n}=\mathcal{Q}_{\lambda}\left[\sum_{\lambda \in B(\lambda)} c(\mu, \lambda) G_{\mu, \lambda} \Delta^{n-|\mu, \lambda|-1} G_{\lambda, \mu}\right] \mathcal{Q}_{\lambda},
$$

where $G_{\mu, \lambda}$ is a product of twistor operators, $c(\mu, \lambda)$ are constants, $G_{\lambda, \mu}$ is the inverse operator of $G_{\mu, \lambda}, \lambda=\left(l_{1}, \ldots, l_{k}\right), \mu=\left(\mu_{1}, \ldots, \mu_{k}\right),|\mu, \lambda|=\sum_{i=1}^{k}\left|\mu_{i}-l_{i}\right|$, and where $B(\lambda)=\left[l_{2}, l_{1}\right] \times\left[l_{3}, l_{2}\right] \times \cdots \times\left[l_{k-1}, l_{k}\right] \times\left[0, l_{k}\right]$. Replacing $\partial_{x}$ by $e_{m}$, we get

$$
(-1)^{n}=p_{\mathfrak{o s p}(1,2 k)}\left[e_{m}\right]\left[\sum_{\lambda \in B(\lambda)} c(\mu, \lambda) G_{\mu, \lambda}\left[e_{m}\right](-1)^{n-|\mu, \lambda|-1} G_{\lambda, \mu}\left[e_{m}\right]\right] p_{\mathfrak{o s p}(1,2 k)}\left[e_{m}\right] .
$$

One can choose $n=l_{1}+1$. This proves that $p_{\mathfrak{o s p}(1,2 k)}\left[e_{m}\right]$ is indeed invertible.

The following corollary is crucial.

Corollary 1. As vector spaces,

$$
\operatorname{ker}_{h} \mathcal{Q}_{\lambda} \cong \mathcal{P}_{h}\left(\mathbb{R}^{m-1}\right) \otimes \mathcal{S}_{\lambda},
$$

Proof. This is true, since the generalised CK-extension tells us that each $f \in \operatorname{ker} \mathcal{Q}_{\lambda}$ is in 1 - 1-correspondence with an $f^{*}\left(x^{*}\right)=f\left(x^{*}, 0\right) \in \mathcal{C}^{\infty}\left(\mathbb{R}^{m-1}, \mathcal{S}_{\lambda}\right)$.

This means that

$$
\operatorname{dim} \operatorname{ker}_{h} \mathcal{Q}_{\lambda}=\operatorname{dim}\left(\mathcal{P}_{h}\left(\mathbb{R}^{m-1}\right)\right) \operatorname{dim}\left(\mathcal{S}_{\lambda}\right) .
$$

Hence, we must find a way to calculate the dimension of $\mathcal{S}_{\lambda}$. 


\subsection{Dimension formula for $\mathcal{S}_{\lambda}$}

In order to calculate the dimension of $\mathcal{S}_{\lambda}$ as a $\operatorname{Spin}(m)$-representation, we make use of the Weyl dimension formula (e.g. [14]), stating that

$$
\operatorname{dim} \mathcal{S}_{\lambda}=\frac{\prod_{\alpha \in \Delta^{+}}\langle\lambda+\delta, \alpha\rangle}{\prod_{\alpha \in \Delta^{+}}\langle\delta, \alpha\rangle},
$$

whereby $\delta=\left(n-\frac{1}{2}, n-\frac{3}{2}, \ldots, \frac{1}{2}\right)$, half the sum of the positive weights, $\langle\cdot, \cdot\rangle$ is the Killing form, and $\Delta^{+}$the positive root system

$$
\begin{array}{r}
\Delta^{+}=\{(1, \pm 1,0, \ldots, 0),(1,0, \pm 1,0, \ldots, 0), \ldots,(0, \ldots, 0,1, \pm 1) \\
(1,0, \ldots, 0),(0,1,0, \ldots, 0), \ldots,(0, \ldots, 0,1)\} .
\end{array}
$$

The denominator of the dimension formula is then given by

$$
\begin{aligned}
\prod_{\alpha \in \Delta^{+}}\langle & \delta, \alpha\rangle \\
= & \left(n-\frac{1}{2}+n-\frac{3}{2}\right)\left(n-\frac{1}{2}+n-\frac{5}{2}\right) \cdots\left(n-\frac{1}{2}+\frac{1}{2}\right) \\
& \times\left(n-\frac{3}{2}+n-\frac{5}{2}\right)\left(n-\frac{3}{2}+n-\frac{7}{2}\right) \cdots\left(n-\frac{3}{2}+\frac{1}{2}\right) \\
& \times \cdots\left(\frac{3}{2}+\frac{1}{2}\right) \\
& \times\left(n-\frac{1}{2}-n+\frac{3}{2}\right)\left(n-\frac{1}{2}-n+\frac{5}{2}\right) \cdots\left(n-\frac{1}{2}-\frac{1}{2}\right) \\
& \times\left(n-\frac{3}{2}-n+\frac{5}{2}\right)\left(n-\frac{3}{2}-n+\frac{7}{2}\right) \cdots\left(n-\frac{3}{2}-\frac{1}{2}\right) \\
& \times \cdots\left(\frac{3}{2}-\frac{1}{2}\right) \\
& \times\left(n-\frac{1}{2}\right)\left(n-\frac{3}{2}\right) \cdots \frac{1}{2},
\end{aligned}
$$

which reduces to

$$
(2 n-2) !(2 n-4) ! \cdots 2 ! \frac{(2 n-1)(2 n-3) \cdots 1}{2^{n}}=\frac{1}{2^{n}} \prod_{j=1}^{n}(2 j-1) ! .
$$

The numerator of (12), is obtained as follows:

$$
\begin{aligned}
& \prod_{\alpha \in \Delta^{+}}\langle\lambda+\delta, \alpha\rangle \\
= & \prod_{1 \leq i<j \leq k}\left(l_{i}+l_{j}+2 n-i-j+2\right)\left(l_{i}-l_{j}+j-i\right) \prod_{j=1}^{k} \frac{\left(l_{i}+2 n-k-i+1\right) !}{\left(l_{i}+k-i\right) !} \prod_{j=1}^{n-k}(2 j-1) ! .
\end{aligned}
$$


This thus leads to

$$
\begin{aligned}
\operatorname{dim}\left(\mathcal{S}_{\lambda}\right)=2^{n} \prod_{1 \leq i<j \leq k}\left(l_{i}+l_{j}+2 n\right. & -i-j+2)\left(l_{i}-l_{j}+j-i\right) \\
& \times \prod_{j=1}^{k}\left(\begin{array}{c}
l_{j}+2 n-k-j+1 \\
2 n-2 k+1
\end{array}\right) \frac{(2 n-2 k+1) !}{(2 n-2 k+2 j-1) !}
\end{aligned}
$$

We can implement it in Maple by means of the code

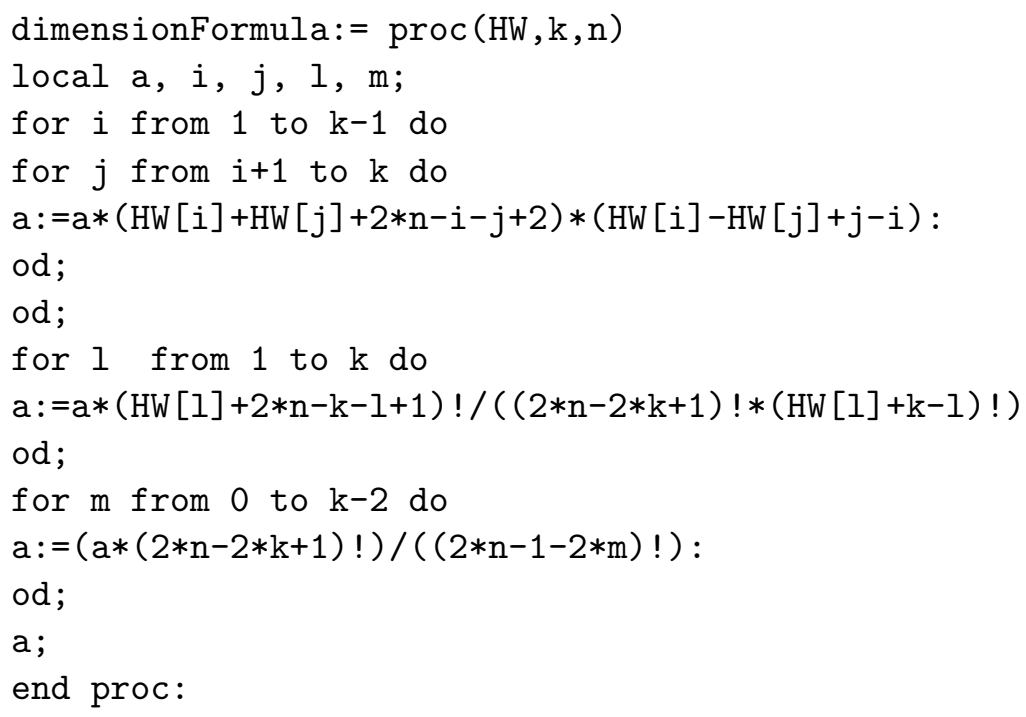

In this code, $H W$ represents $\lambda$ as a $(1 \times k)$-matrix.

\subsection{Open problem}

We can use formula (13) from the previous subsection to finish the proof of Proposition 4. Indeed, on the one hand side, we have that

$$
\mathcal{K}_{h ; \lambda} \cong \bigoplus_{j_{1}=0}^{l_{1}-l_{2}} \cdots \bigoplus_{j_{k-1}=0}^{l_{k-1}-l_{k}} \bigoplus_{j_{k}=0}^{l_{k}} \mathcal{K}_{h ; \lambda}^{\left(j_{1}, j_{2}, \ldots, j_{k}\right)} \subseteq \bigoplus_{j_{1}=0}^{l_{1}-l_{2}} \cdots \bigoplus_{j_{k-1}=0}^{l_{k-1}-l_{k}} \bigoplus_{j_{k}=0}^{l_{k}} \mathcal{M}_{h-\sum_{p=1}^{k} j_{p} ; l_{1}-j_{1}, \ldots, l_{k}-j_{k}}^{s}
$$

due to (11). If we can prove that the dimensions of the spaces on both sides of the equation are equal, then the proof is finished. Due to Corollary 1, we have:

$$
\operatorname{dim}\left(\mathcal{K}_{h ; \lambda}\right)=\operatorname{dim}\left(\mathcal{P}_{h}\left(\mathbb{R}^{m-1}\right)\right) \operatorname{dim}\left(\mathcal{S}_{\lambda}\right) .
$$


On the other hand, thanks to Theorem 1, we have that the the dimension of the space on the righthandside equals

$$
\begin{aligned}
& \operatorname{dim}\left(\bigoplus_{j_{1}=0}^{l_{1}-l_{2}} \cdots \bigoplus_{j_{k-1}=0}^{l_{k-1}-l_{k}} \bigoplus_{j_{k}=0}^{l_{k}} \mathcal{M}_{h-\sum_{p=1}^{k} j_{p} ; l_{1}-j_{1}, \ldots, l_{k}-j_{k}}^{s}\right) \\
& =\sum_{j_{1}=0}^{l_{1}-l_{2}} \cdots \sum_{j_{k-1}=0}^{l_{k-1}-l_{k}} \sum_{j_{k}=0}^{l_{k}} \sum_{i_{1}=0}^{l_{1}-j_{1}-l_{2}+j_{2}} \\
& \ldots \sum_{i_{k-1}=0}^{l_{k-1}-j_{k-1}-l_{k}+j_{k}} \sum_{i_{k}=0}^{l_{k}-j_{k}} \operatorname{dim}\left(\mathcal{S}_{h+\sum_{p=1}^{k} i_{p}-j_{p}, l_{1}-i_{1}-j_{1}, \ldots, l_{k}-i_{p}-j_{p}}\right) .
\end{aligned}
$$

\begin{tabular}{|c|c|}
\hline$k$ & $\operatorname{dim} \mathcal{K}_{h ; \lambda}$ \\
\hline 1 & $2^{n}\left(\begin{array}{c}h+2 n-1 \\
h\end{array}\right)\left(\begin{array}{c}l_{1}+2 n-1 \\
l_{1}\end{array}\right)$ \\
\hline 2 & $2^{n}\left(\begin{array}{c}h+2 n-1 \\
h\end{array}\right)\left(\begin{array}{c}l_{1}+2 n-2 \\
l_{1}+1\end{array}\right)\left(\begin{array}{c}l_{2}+2 n-3 \\
l_{2}\end{array}\right) \frac{\left(l_{1}+l_{2}+2 n-1\right)\left(l_{1}-l_{2}+1\right)}{(2 n-1)(2 n-2)}$ \\
\hline 3 & $\begin{array}{c}2^{n}\left(\begin{array}{c}h+2 n-1 \\
h\end{array}\right)\left(\begin{array}{c}l_{1}+2 n-3 \\
l_{1}+2\end{array}\right)\left(\begin{array}{l}l_{2}+2 n-4 \\
l_{2}+1\end{array}\right)\left(\begin{array}{c}l_{3}+2 n-5 \\
l_{3}\end{array}\right) \\
\times \frac{\left(l_{1}+l_{2}+2 n-1\right)\left(l_{1}+l_{3}+2 n-2\right)\left(l_{2}+l_{3}+2 n-3\right)\left(l_{1}-l_{2}+1\right)\left(l_{1}-l_{3}+2\right)\left(l_{2}-l_{3}+1\right)}{(2 n-1)(2 n-2)(2 n-3)^{2}(2 n-4)^{2}}\end{array}$ \\
\hline
\end{tabular}

In the case of $k=1,2$ and 3 this is symbolically doable with Maple [21] using the code above, and the respective dimensions are indeed found to be equal:

However, in full generality this still needs to be confirmed.

\section{Conclusion}

In this paper, we found a way to decompose the $\operatorname{space} \operatorname{ker}_{h} \mathcal{Q}_{\lambda}$ of null solutions for an arbitrary HSD operator using an inductive procedure (exploiting the power of the twistor operators and the twisted version of the HSD operators). Invoking the CK-extension, this reduced the problem to a combinatorial counting argument. For the cases $k \in\{1,2,3\}$ (the number of dummy variables describing the values of our higher spin fields) this was verified explicitly, whereas the general case seems to be out of grasp at this point.

\section{References}

[1] Brackx, F., Delanghe, R., Sommen, F., Clifford Analysis, Research Notes in Mathematics 76, Pitman, London, 1982.

[2] Brackx, F., Eelbode, D., Van de Voorde, L., The polynomial null solutions of a higher spin Dirac operator in two vector variables, Adv. Appl. Cliff. Alg. 21 No. 3 (2011), pp. $455-476$.

[3] Brackx, F., Eelbode, D., Van de Voorde, L., Higher spin Dirac operators between spaces of simplicial monogenics in two vector variables, J. Math. Phys. Anal. Geom. (2011) $14: 1-20$ 
[4] Branson, T., Stein-Weiss operators and ellipticity, J. Funct. Anal. 151 No. 2 (1997), pp. 334-383.

[5] Bureš, J., Sommen, F., Souček, V., Van Lancker, P., Rarita-Schwinger type operators in Clifford analysis, Journal of Funct. Anal. 185, pp. 425-456.

[6] Bureš, J., Sommen, F., Souček, V., Van Lancker, P., Symmetric analogues of RaritaSchwinger equations, Ann. Glob. Anal. Geom. 21 No. 3 (2001), pp. 215-240.

[7] Constales, D., Sommen. F., Van Lancker, P., Models for irreducible representations of Spin(m), Adv. Appl. Clifford Algebras 11 No. S1 (2001), pp. 271-289.

[8] Eelbode, D., Smid, D., Factorization of Laplace operators on higher spin representations, to appear in Compl. Anal. Oper. Theo.

[9] De Schepper, H., Eelbode, D., Raeymaekers, T., On a special type of solutions for arbitrary higher spin Dirac Operators, J. Phys. A: Math. Theor. 43 No. 32 (2010) pp. $1-13$.

[10] De Schepper, H., Eelbode, D., Raeymaekers, T., Twisted higher spin Dirac operators, submitted.

[11] Delanghe, R., Sommen, F., Souček, V., Clifford analysis and spinor valued functions, Kluwer Academic Publishers, Dordrecht, 1992.

[12] Eelbode, D., Raeymaekers, T., Construction of higher spin operators using transvector algebras, submitted

[13] Fegan, H. D., Conformally invariant first order differential operators, Quart. J. Math. 27 (1976), pp. 513-538.

[14] Fulton, W., Harris, J., Representation theory: a first course, Springer-Verlag, New York, 1991.

[15] Gilbert, J., Murray, M.A.M., Clifford algebras and Dirac operators in harmonic analysis, Cambridge University Press, Cambridge, 1991.

[16] Guerlebeck, K., Sprossig, W., Quaternionic and Clifford Calculus for Physicists and Engineers, Wiley, 1998.

[17] Howe, R., Transcending classical invariant theory, J. Amer. Math. Soc. 2 No. 3 (1989), pp. 535-552.

[18] Howe, R., Remarks on classical invariant theory, Trans. Amer. Math. Soc. 313 No. 2 (1989), pp. 539-570.

[19] Humphreys, J., Introduction to Lie algebra and representation theory, SpringerVerlag, New York, 1972. 
[20] Klimyk, A. U., Infinitesimal operators for representations of complex Lie groups and Clebsch-Gordan coefficients for compact groups, J. Phys. A: Math. Gen 15 (1982), pp. 3009-3023.

[21] Maple 17, Maplesoft, a division of Waterloo Maple Inc., Waterloo, Ontario.

[22] Molev, A.I., Yangians and classical Lie algebras, Mathematical surveys and monographs 143, AMS Bookstore (2007).

[23] Rarita, W., Schwinger, J., On a theory of particles with half-integer spin, Phys. Rev. 60 (1941), pp. 61.

[24] Slovak, J., Natural operators on conformal manifolds, Masaryk University Dissertation (Brno, 1993)

[25] Stein, E.W., Weiss, G., Generalization of the Cauchy-Riemann equations and representations of the rotation group, Amer. J. Math. 90 (1968), pp. 163-196.

[26] Tolstoy, V.N., Extremal projections for reductive classical Lie superalgebras with a non-degenerate generalised Killing form, Russ. Math. Surv. 40, pp. 241-242, 1985.

[27] Zhelobenko, D.P. Transvector algebras in representation theory and dynamic symmetry, Group Theoretical Methods in Physics: Proceedings of the Third Yurmala Seminar 1, 1985. 